\title{
Change Detection for Thematic Mapping by Means of Airborne Multitemporal Polarimetric SAR Imagery
}

Dierking, W.; Skriver, Henning

Published in:

I E E E Transactions on Geoscience and Remote Sensing

Link to article, DOI:

10.1109/TGRS.2002.1000322

Publication date:

2002

Document Version

Publisher's PDF, also known as Version of record

Link back to DTU Orbit

Citation (APA):

Dierking, W., \& Skriver, H. (2002). Change Detection for Thematic Mapping by Means of Airborne Multitemporal Polarimetric SAR Imagery. I E E E Transactions on Geoscience and Remote Sensing, 40(3), 618-636. https://doi.org/10.1109/TGRS.2002.1000322

\section{General rights}

Copyright and moral rights for the publications made accessible in the public portal are retained by the authors and/or other copyright owners and it is a condition of accessing publications that users recognise and abide by the legal requirements associated with these rights.

- Users may download and print one copy of any publication from the public portal for the purpose of private study or research.

- You may not further distribute the material or use it for any profit-making activity or commercial gain

- You may freely distribute the URL identifying the publication in the public portal 


\title{
Change Detection for Thematic Mapping by Means of Airborne Multitemporal Polarimetric SAR Imagery
}

\author{
Wolfgang Dierking and Henning Skriver
}

\begin{abstract}
The paper addresses the detection of changes in multitemporal polarimetric radar images, focusing on small objects and narrow linear features. The images were acquired at $\mathrm{C}$ - and L-band by the airborne EMISAR system. It is found that the radar intensities are better suited for change detection than the correlation coefficient and the phase difference between the co-polarized channels. In the case of linear features, there is no obvious difference between $C$ - and L-band, and slight variations of the flight tracks are acceptable at look angles larger than 35 degrees. Theoretical detection thresholds are evaluated from the statistical distribution of the intensity ratio due to speckle. For the linear features and for urban environments, the observed thresholds are larger than the theoretical predictions. This is interpreted as an effect of radar intensity variations on length scales smaller than the spatial image resolution. The signature of urban areas is very sensitive to deviations between the flight tracks, and the sensitivity is larger at C-band than at L-band. On the other hand, the intensity contrast between buildings and the urban background is smaller at $\mathrm{L}$-band and larger at $\mathrm{C}$-band. For change detection, thresholds may have to be chosen separately for each object class because the intensity ratios of different object classes vary differently as a function of time.
\end{abstract}

Index Terms-Change detection, polarimetry, synthetic aperture radar (SAR).

\section{INTRODUCTION}

D URING the last decade, the problem of change detection by means of synthetic aperture radar (SAR) intensity images has been treated by several authors with focus on different aspects. The reduction of speckle and the co-registration of the multitemporal images were pointed out as major topics for the development of a successful detection scheme [1]-[4]. Instead of working with data representing the difference of two images as it is usually done in optical remote sensing, the ratio between two intensity images is preferable because of the statistical characteristics of radar data [5], [6]. The utilization of SAR images in change detection was assessed in the case of both spaceborne [3]-[7] and airborne sensors [1], [2], focusing on different land cover classes such as agricultural environments, forests, and urban areas. Specifically Weydahl [7] dealt with the identification of small objects (i.e., objects covering only a few pixels) in ERS-1 scenes with the aid of SPOT and Landsat TM images, and discussed examples where physical changes of these objects were reflected in the SAR data.

Manuscript received March 29, 2001; revised December 4, 2001. This work was supported by the Danish Research Councils under the ESA Follow-On Research Program for the TOPOSAR Project.

The authors are with the Department of Electromagnetic Systems, Technical University of Denmark, DK-2800 Lyngby, Denmark (e-mail: wd@emi.dtu.dk). Publisher Item Identifier S 0196-2892(02)04354-1.
The optimal prerequisite for change detection by means of SAR is that the images to be compared are measured with the same sensor setup (same frequency, polarization, and illumination geometry) and generated with identical processing parameters. In practice, small differences between the flight tracks cause slight changes of the illumination geometry and, hence, variations in the signatures. Other technical problems, which might contribute to the observed signature variability, are errors in the image calibration, and inaccuracies of the image co-registration. Even if the technical effects are negligible, several objects of interest reveal inherent temporal signature changes, which are not subject of map updates. Intuitively this is to be expected, for example, for many types of natural vegetation (e.g., [2], [8]). The reason is that the dielectric properties and the scattering characteristics of many natural objects change as a function of time.

The objective of this work is to assess the possibility of utilizing multitemporal polarimetric images for change detection of object classes which are relevant for updating thematic maps. Small objects (such as buildings) and narrow linear features (such as roads) are considered which usually are of great interest to the mapping agencies. The paper deals specifically with two items: Firstly, it is studied how useful different polarimetric parameters and radar frequency bands are with regard to the detection of changes. Secondly, the separation of signature variations due to man-made changes (in most cases the addition or removal of an object to the scene), on the one hand, and due to speckle and natural scattering variations (as observed for vegetation, for example), on the other hand, is addressed. Airborne SAR data are used which have a better spatial resolution than spaceborne data and hence are better suited for the utilization in thematic mapping. For the project, high-resolution aerial photographs were available which made it easier to identify even very small objects in the polarimetric images.

The data used in this work and their preparation for the analysis are described in Section II. The section also includes an assessment of the relative calibration accuracy and comments the choice of the object classes. In Section III, the average signatures and the spatial signature variations of the different object classes are evaluated. The signature averages are the starting point for analyzing the image-to-image signature stability of each of the object classes as well as their signature contrast relative to one another. The results presented in Section III reveal also the contributions of natural variations of the radar signature in comparison to the contribution of speckle. The usefulness of the correlation coefficient and the phase difference between the co-polarized SAR channels is discussed. Section IV deals with 
TABLE I

\begin{tabular}{|c|c|}
\hline Location & Denmark, Jutland, around Research Center Foulum \\
\hline Image type & fully polarimetric \\
\hline Bands (frequency, wavelength) & $C(5.3 \mathrm{GHz}, 0.057 \mathrm{~m})$ and $L(1.25 \mathrm{GHz}, 0.24 \mathrm{~m})$ \\
\hline Incidence angle range & $35-60$ deg \\
\hline Heading (flight direction) & $117 \mathrm{deg}$ \\
\hline Pixel spacing & $5 m$ \\
\hline Effective spatial resolution & $8 m$ \\
\hline Number of looks & 11 (C-band), 9 (L-band) \\
\hline $\begin{array}{l}\text { Backscattering coefficient } \\
\text { equivalent to noise level at mid- } \\
\text { range }\end{array}$ & $38 d B(C-$ band $)$, and $47 d B(L-b a n d)$ \\
\hline $\begin{array}{l}\text { Time of acquisition } \\
\text { (in parentheses, number of data } \\
\text { acquisitions in the given period) }\end{array}$ & $\begin{array}{l}\text { March } 98(2), \\
\text { April } 98 \text { - August } 98(5, \text { time interval about I month }) \text {, } \\
\text { June } 99 \text { (L-band only) }\end{array}$ \\
\hline $\begin{array}{l}\text { Differences of incidence angles } \\
\text { at a given position in the scene }\end{array}$ & $\begin{array}{l}\text { reference incidence angle } 35 \mathrm{deg} \text { (April, May, July, August 98) } \\
29.2 \mathrm{deg} \text { (March 98), } 34 \mathrm{deg} \text { (June 98) } \\
\text { reference angle } 60 \mathrm{deg} \text { (April, May, July, August 98) } \\
57.8 \mathrm{deg} \text { (March 98), } 59 \mathrm{deg} \text { (June 98) }\end{array}$ \\
\hline Analyzed parameters & $\sigma_{V V}, \sigma_{H H}, \sigma_{H V}, \rho_{H H V V}, \phi_{H H V}$ \\
\hline
\end{tabular}

temporal signature changes of each object class. A theoretical model of the statistical distribution of the intensity ratio is utilized in order to understand and to predict the differences observed between the individual SAR image pairs. Thresholds are determined from the data so that only a certain amount of inherent temporal variations (due to speckle and natural signature variations) are classified as real changes. These thresholds are compared to the ones predicted theoretically. The outcome of the study in a broader context is discussed in Section V, and finally, the summary and conclusions are presented in Section VI.

\section{METHODOLOGY}

\section{A. Data Preparation}

The images used in this project were acquired by the Danish airborne EMISAR (a detailed description of the EMISAR can be found in [9]). A summary of the image properties is given in Table I. The test site is located in Jutland, Denmark, around the Research Center Foulum. The imaged area is a typical agricultural environment, consisting of agricultural fields, farm buildings, and villages with detached houses and small industrial complexes. The most prominent linear features in the radar scenes are tree hedges which form obstacles for the wind and reduce erosion (see Fig. 1).

The data were acquired simultaneously at C- and L-band. The image sequence covers a time interval of five months from
March to August 1998. At L-band, an additional image from June 1999 was available for this study. The nominal flight headings were identical for all flights. The local incidence angles at fixed ground range positions, however, were slightly different due to variations of the flight tracks. The images from April, May, July and August 1998, and June 1999 were measured with the same illumination geometry and are used as references. Relative to the reference flight track, the two tracks flown in March were shifted horizontally, and the track in June was flown at a larger altitude. At reference incidence angles of $35 \mathrm{deg}$ and 60 $\mathrm{deg}$, the deviations for the June 98 scenes are about $1 \mathrm{deg}$, and for the March scenes, the deviations are $5.8 \mathrm{deg}$ at $35 \mathrm{deg}$ and $2.2 \mathrm{deg}$ at $60 \mathrm{deg}$.

The images are in the covariance matrix representation which is an operationally provided EMISAR data product. The covariance matrix elements are given in ground range projection with a pixel spacing of 5 by $5 \mathrm{~m}$. The data are low-pass filtered such that the effective number of independent looks is constant over ground range. Therefore, the correlation length between neighboring pixels decreases, and the dimension of the effective spatial resolution cell increases from the near- to the far-range side of the image. From the variance-to-squared-mean ratio (VMR) of large homogeneous areas within the images, the effective numbers of looks were estimated. These are $L \approx 11$ at $\mathrm{C}$-band and $L \approx 9$ at L-band, the difference is due to different data acquisition and processing parameters. The effective spatial res- 


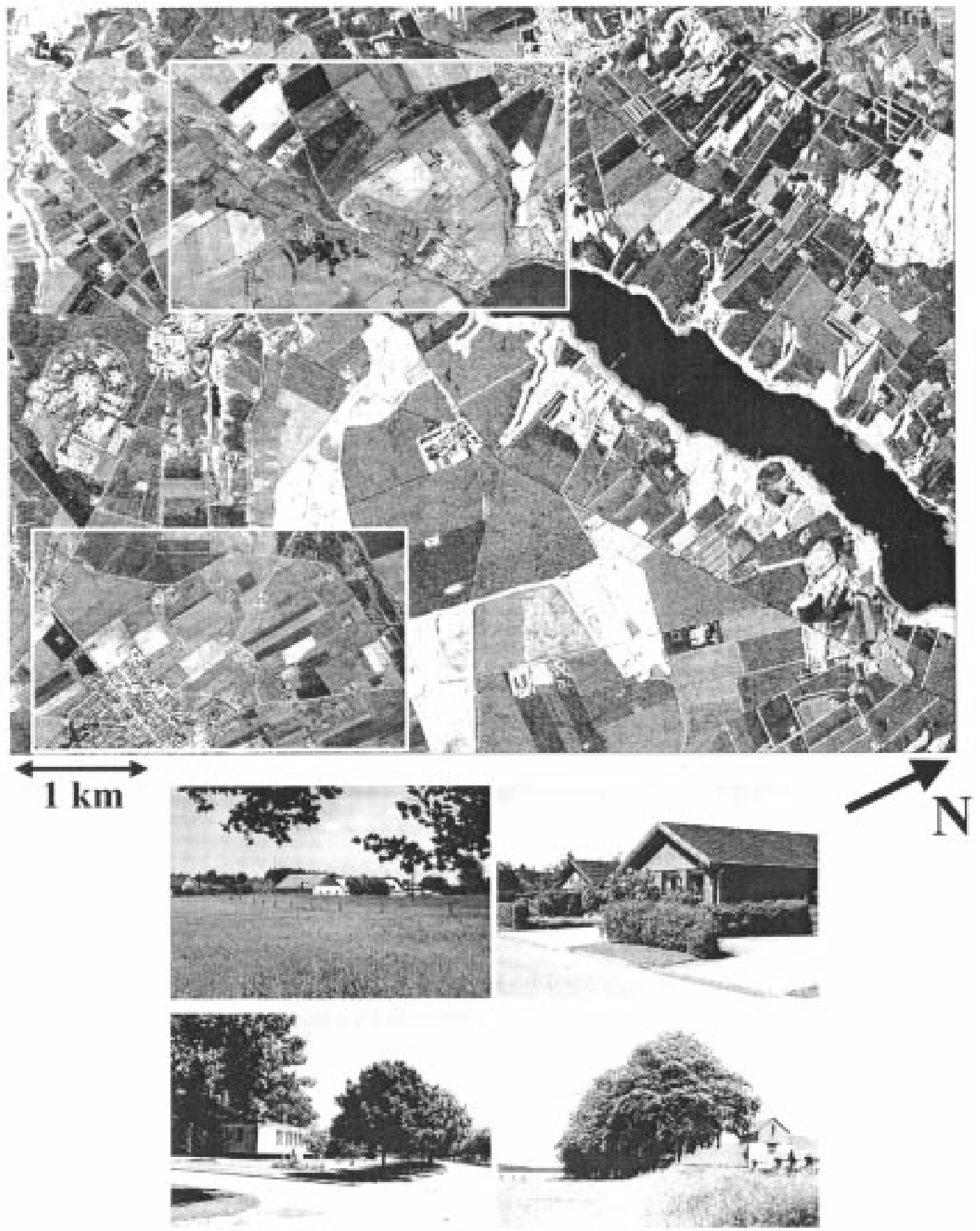

Fig. 1. Radar image over a region within the test site which is a black-and-white representation of an RGB image showing the intensities at HV-, HH-, and VV-polarization. The two smaller boxes show parts of an aerial photograph from the same area. Most of the prominent bright lines in the radar image are tree hedges (shown in the lowermost two in-situ photographs), whereas roads appear as dark lines. Radar images were geometrically corrected using a DEM. They and the aerial photographs were registered to an internal radar mapping system. The test sites consists of agricultural fields and farm houses (in-situ photographs, upper row, left), and small villages with detached houses (in-situ photographs, upper row, right).

olution is roughly $8 \mathrm{~m}$ at mid-range. The speckle noise is sufficiently reduced for the identification of smaller objects in the image.

From the covariance matrix elements, the backscattering coefficients $\sigma_{\mathrm{VV}}^{0}, \sigma_{\mathrm{HV}}^{0}, \sigma_{\mathrm{HH}}^{0}$, and the correlation coefficient between the co-polarized channels (magnitude $\rho_{\mathrm{HHVV}}$ and phase difference $\phi_{\mathrm{HHVV}}$ ) are calculated (see, e.g., [8] for details) and used in subsequent analyzes. The polarization is indicated by the indices, where "V" denotes vertically, and " $\mathrm{H}$ " horizontally polarized radar waves. Only the correlation between the co-polarized channels $\mathrm{VV}$ and $\mathrm{HH}$ is considered, since the correlation between cross- and co-polarized channels (e.g., HH and HV or $\mathrm{VV}$ and $\mathrm{VH}$ ) is very low for many targets at L-band, and can be neglected at C-Band [10, Figs. 11.1 and 11.2]. 
Dealing with objects only a few pixels in size may require adaptive speckle filters which preserve more or less the fine structural details in the radar image, e.g., [10], [11]. A number of sophisticated speckle reduction techniques is available which have to be considered in devising a robust change detection scheme for practical use. However, they may alter the signature mean and statistics in different manners, and they require relative long execution times on the computer. In order to get results not biased by a particular speckle reduction technique, the data used in this study were not filtered further than the usually provided operational image products.

All radar images and aerial photographs were registered to an internal radar mapping coordinate system [12] whereby the registration procedure included the correction of geometric and radiometric distortions using a digital elevation model (DEM). The DEM was generated from interferometric EMISAR data acquired in 1997. Although the registration to a DEM is not necessary for change detection (since all radar data were acquired at an almost identical imaging geometry), it was nevertheless carried out in order to combine the polarimetric images directly with aerial photographs, topographic maps, and thematic vector layers representing different object classes. The radar images were registered to one another with an r.m.s.-accuracy of better than one pixel [12]. Nearest neighbor interpolation was used in the process of resampling. Although, in general, strong aliasing and blurring effects are associated with the nearest neighbor method (e.g., [13]), it was regarded the optimal interpolation scheme for the data available in this study. The reasons are that

- the spatial resolution in the DEM data and the polarimetric images are identical,

- and the angles between the interferometric and polarimetric flight tracks are very small.

Hence, only a small number of pixels present in the original image was omitted or used twice in the resampled image. On the other hand, the effective spatial resolution was not reduced which was essential for the preservation of small details in the resampled scenes.

\section{B. Image Calibration}

For a set of selected EMISAR scenes acquired at identical illumination geometries, Christensen et al. [9] examined the map-to-map calibration stability and found that it was $\pm 0.1 \mathrm{~dB}$ at C-band and $\pm 0.5 \mathrm{~dB}$ at L-band (standard deviation, averaged over range). Unpublished tests by Dierking revealed that the peak-to-peak variations at single calibration targets are on the order of $\pm 1 \mathrm{~dB}$ at C-band and $\pm 1.5 \mathrm{~dB}$ at L-band for the measured intensities. The corresponding variations of the phase difference, $\phi_{\text {HHVV }}$, between the like-polarized channels is $\pm 5 \mathrm{deg}$ at C-band and \pm 10 deg at L-band. These tests were carried out using several data sets which were acquired with different illumination geometries so that the local incidence angles at the positions of the calibration targets were different.

In order to assess the calibration stability of the images used in this study, the average signature of forests obtained from the different images were compared as a function of the local incidence angle. For incidence angle intervals of $5 \mathrm{deg}$ in width, all pixels belonging to marked forest areas were pooled, and mean and variance were calculated. The forests used for checking the calibration stability consist predominantly of coniferous trees which reveal only small signature changes in the period from April to August.

The example shown in Fig. 2(a) is the backscattering coefficient at VV-polarization. It should be noted again that the same areas were imaged with slightly different incidence angles which is reflected in Fig. 2(a) and also in the following plots. The deviations between the intensities at VV-polarization measured over forest in the different images are at maximum about $2 \mathrm{~dB}$, that is, they are well within the calibration tests mentioned above. A peculiarity at L-band is the large difference of the average intensities between the two data acquisitions in March. The processing log of the data was carefully examined, in particular with regard to calibration, but an explanation for this difference could not be found.

The temporal variations of the average phase difference between the $\mathrm{HH}$ - and $\mathrm{VV}$-polarized channels are depicted in Fig. 2(b). At C-band, the maximum observed deviation between single images is slightly larger than $15 \mathrm{deg}$. At L-band, the corresponding value is less than $10 \mathrm{deg}$, with the exception of incidence angles $>52 \mathrm{deg}$ where the phase differences from March differ more clearly from those of the other months. In comparison to the calibration tests mentioned above, there is again no significant difference.

\section{Object Classes}

In this work, three object classes are considered, namely buildings, roads, and tree hedges which are examples of objects regularly monitored by local authorities. These objects are comparatively small or narrow in the radar images. Buildings comprise only a few pixels, and roads and hedgerows are only 1-3 pixels wide. Hence, these objects are often difficult to detect using radar images alone. Pixels in the radar images belonging to certain objects were identified by comparison with aerial photography. Straight line segments on roads and hedgerows as well as pixels covering buildings were marked. Usually, small or linear objects are surrounded by extended targets such as vegetated surfaces. For detecting changes of small and linear objects, the signature characteristics of neighboring extended targets may have to be considered as well. Investigations of temporal changes of extended targets can be found, e.g., in [2], [5]-[8]. In this study, only forest areas and the urban background (which is the area within a village not covered by buildings) were included as additional object classes, the former as sort of a reference. Information about the signature characteristics of the urban background is (to the authors' knowledge) not or only sparsely available.

\section{SignATURE Characteristics OF DifFERENT OBJeCt CLASSES}

There are two major factors which contribute to the performance of any change detection technique applied to SAR data:

- the inherent temporal signature variability of an object class due to speckle and due to natural variations of the physical characteristics of the imaged scene, 
Forest

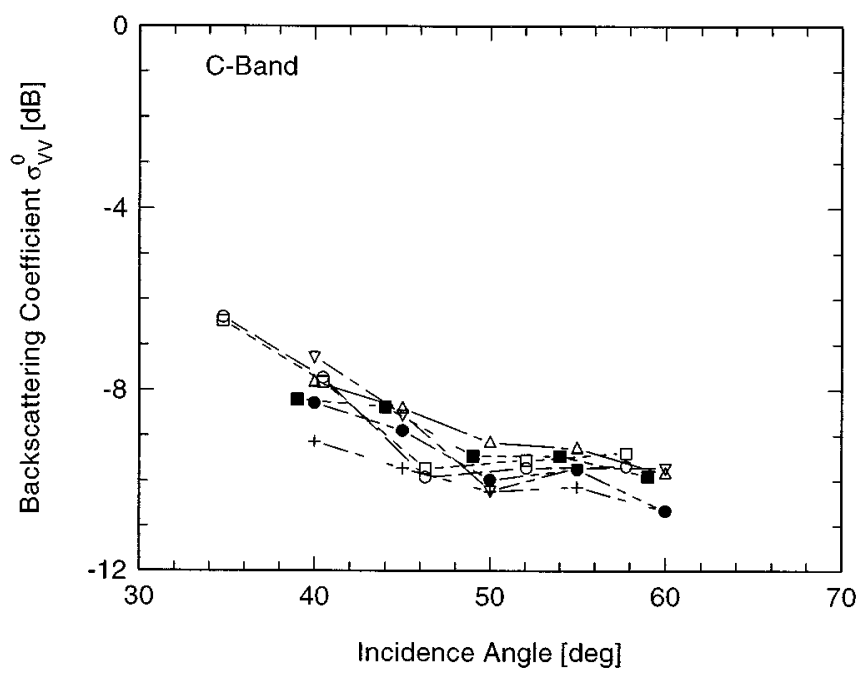

Forest

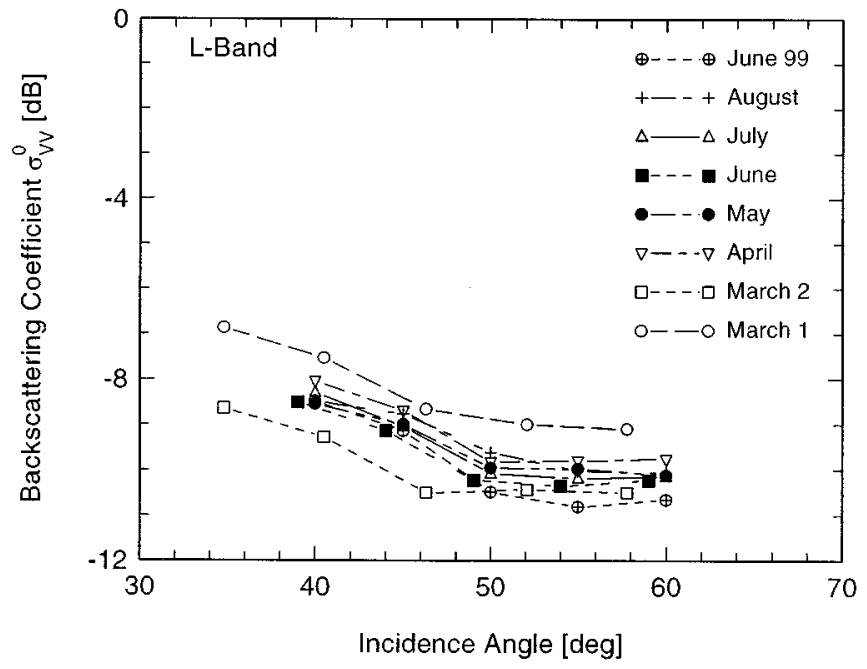

(a)

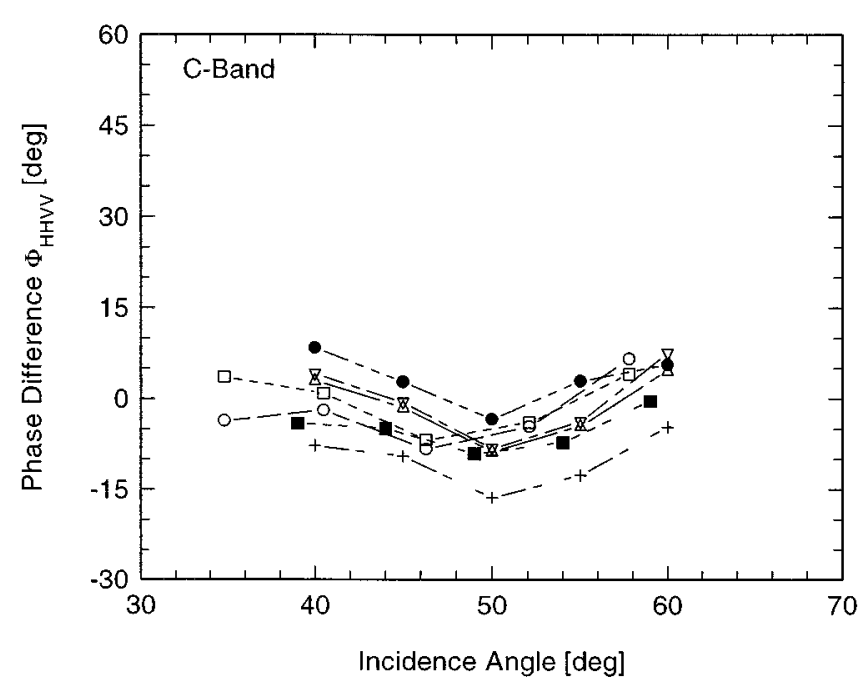

Forest

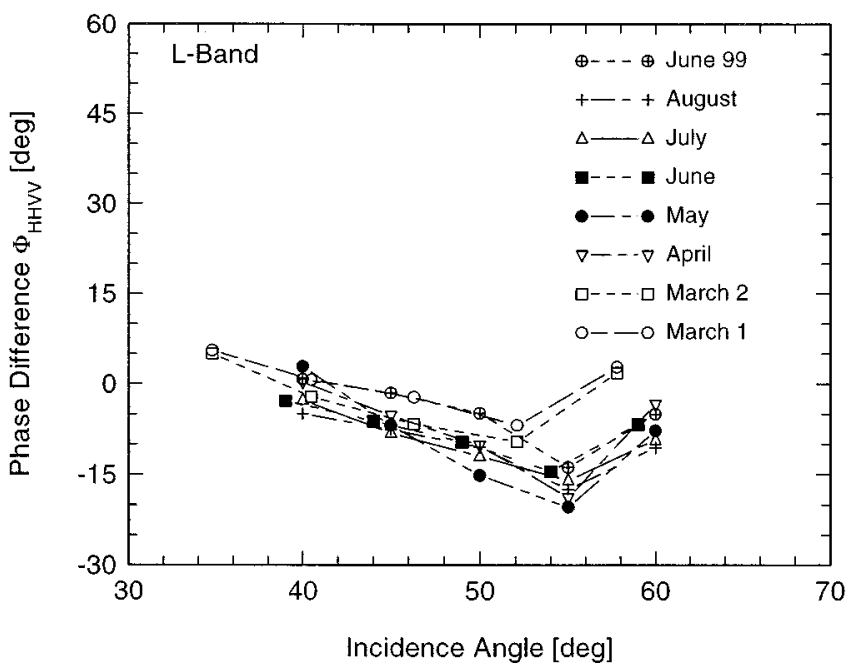

(b)

Fig. 2. (a) Backscattering coefficients of forest at C- and L-band, VV-polarization, evaluated for different ground range intervals from images acquired at different times. Note that the radar signatures were determined for the same areas in all images. The number of averaged pixels for each area is larger than 28000 (except for the near-range area, where the number of pixels is 2560), so that the effect of speckle can be neglected. (b) Phase differences between HH- and VV-polarized signals of forest at C- and L-band, evaluated in parallel to the backscattering coefficients shown in Fig. 2(a).

- the contrast of the average signature between an object and its background or another object which replaces the first one.

The signature contrast may determine whether or not the adding or the removal of a smaller object can be recognized in the radar image. A road, e.g., may be built through a vegetated area. The road is recognized as a change in a radar image pair if its signature is different compared to the vegetation (the background). On the other hand, if, e.g., a building is pulled down, the surrounding vegetation will not necessarily spread out during the time span between data acquisitions over the place where the building was located.

In this section, the average signatures and the spatial signature variance of the different object classes are evaluated for all used SAR scenes. Inter-comparisons of the average values of the different polarimetric parameters are helpful in order to as- sess the temporal signature stability and object class contrasts. Natural signature variations within one scene relative to speckle are examined.

\section{A. Average Signatures}

The mean signatures of the different object classes, plotted for each acquired data set, are a useful indicator of general large scale changes. Since a large number of pixels is averaged, the influence of speckle is negligible or at least very small.

The average intensity variations of hedges as a function of the incidence angle, shown in Fig. 3(a), are slightly larger than those of forests. This can also be recognized by eye in the radar images where a number of hedges show temporal signature variations. Except for small incidence angles at C-band (March data), there is no significant decrease of the backscattered intensity as a function of incidence angle which indicates that volume scat- 
Hedges

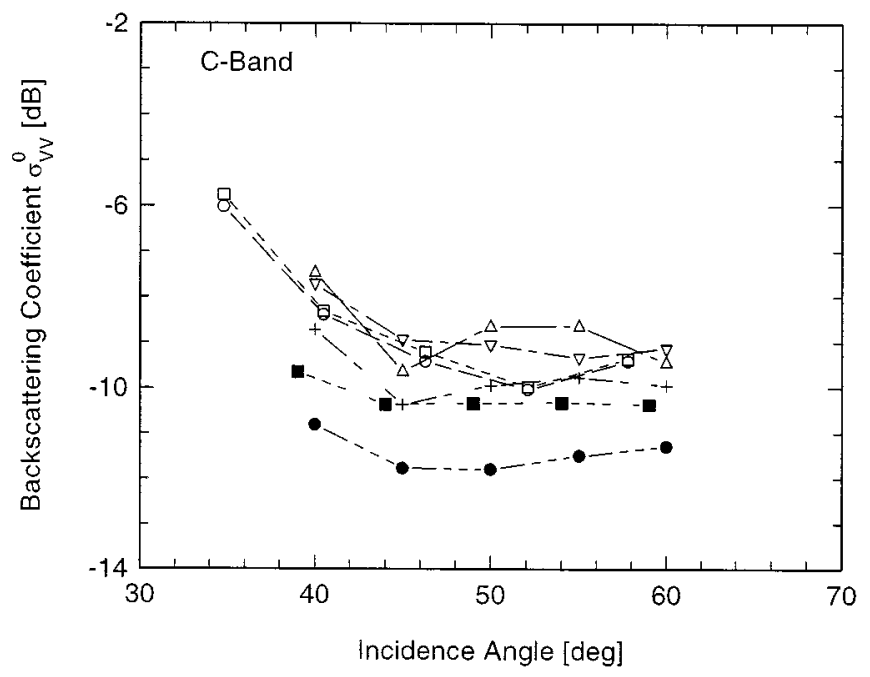

Hedges

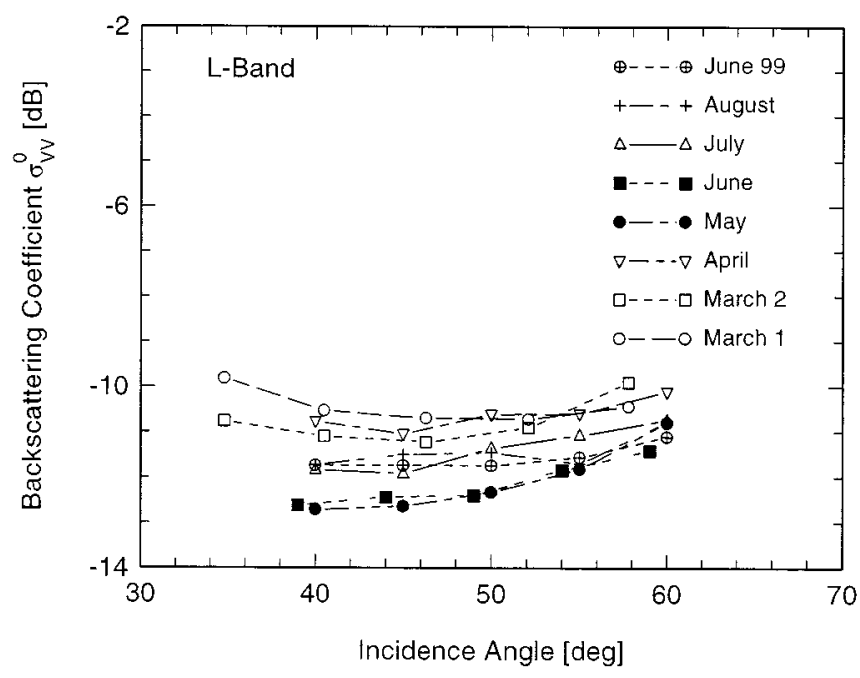

(a)

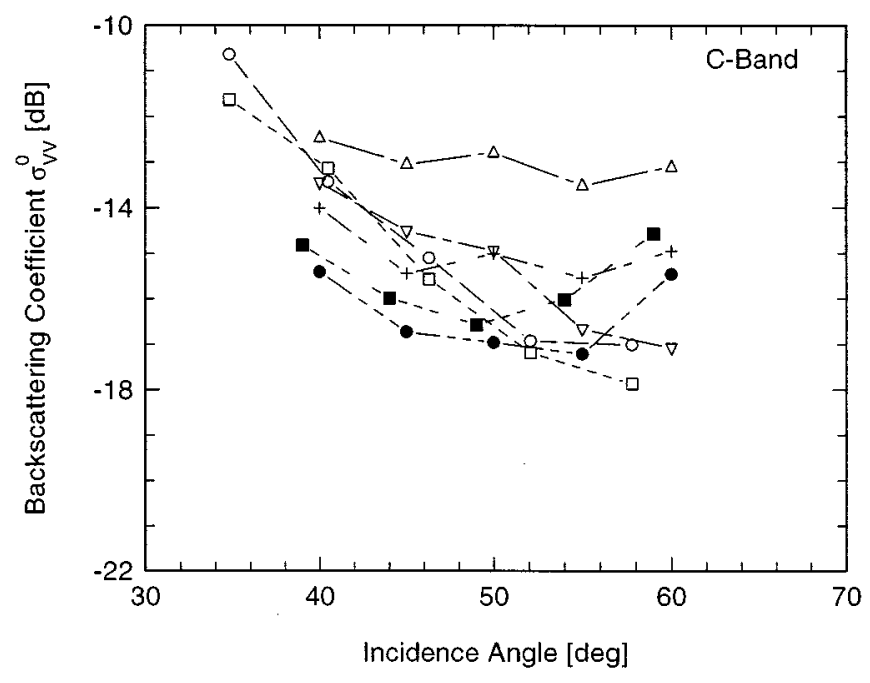

Roads

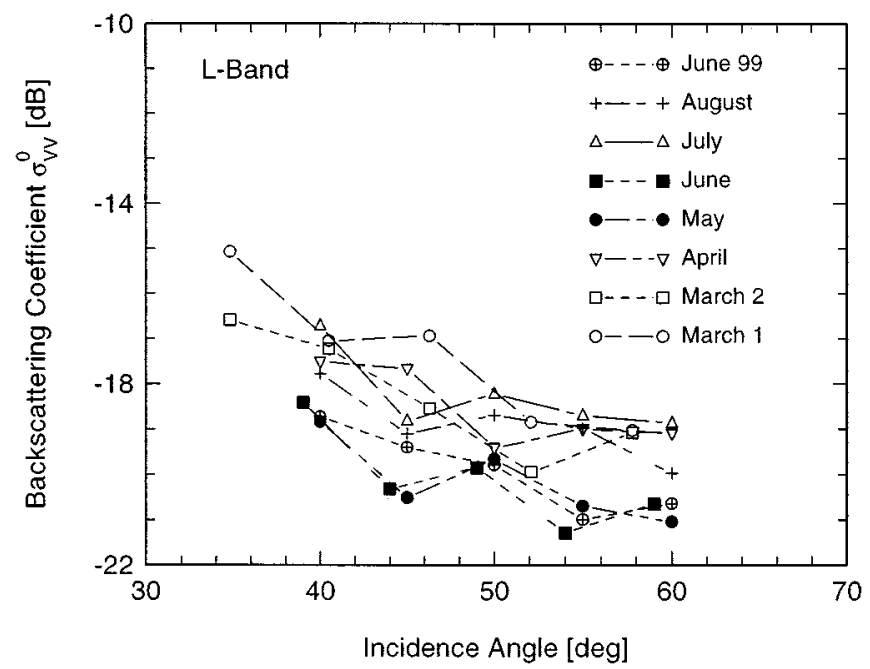

(b)

Fig. 3. (a) Backscattering coefficients of hedges at C- and L-band, VV-polarization. The number of averaged pixels is larger than 900. (b) Backscattering coefficients of roads at C- and L-band, VV-polarization. The number of averaged pixels is larger than 170 so that the effect of speckle is small.

tering is dominant at larger incidence angles. This is consistent with a relatively large cross-polarization intensity which lies between $-17.3 \mathrm{~dB}$ and $-13.3 \mathrm{~dB}$ at C-band, and $-14.3 \mathrm{~dB}$ and $-18.1 \mathrm{~dB}$ at $\mathrm{L}$-band in the incidence angle range between 35 and 60 deg. The temporal signature evolution shown in Fig. 3(a) does not indicate any clear correlation with the season, i.e., with the development of the leaves.

Roads reveal a significantly lower average intensity and a slightly larger signature variation between the different images than forests or hedges [Fig. 3(b)]. The general tendency of a decreasing intensity with increasing incidence angle is expected for surface scattering. At C-band, however, the intensities measured in May and June increase at larger incidence angles. In the interpretation of the signatures, it has to be considered that the roads at the test site are relatively narrow. Several pixels contain signature contributions of the vegetation adjacent to the road. The physical characteristics of the vegetation change from month to month which is reflected in the observed signature variations of the class "roads." This interpretation is supported by the observation that the signature difference is small between June 1998 and June 1999 [Fig. 3(b)].

The backscattering coefficient of buildings varies much more [Fig. 3(c)], and it does not show any clear correlation with the incidence angle, $\theta$, which is not surprising. The backscattered intensity observed at a certain radar incidence angle depends on the occurrence of specular facets, dihedrals or other structural elements as part of the buildings and the urban background, and on their relative orientation to the radar. Slight variations of the radar imaging geometry, i.e., of the incidence and azimuth angle, might cause considerable changes of the measured signature. That explains why the signature variation of buildings, which are stable objects, can be larger than the signature variation of more changeable object such as hedges. At C-band, the shape of the curves $\sigma_{\mathrm{VV}}^{0}(\theta)$ obtained for the different data ac- 

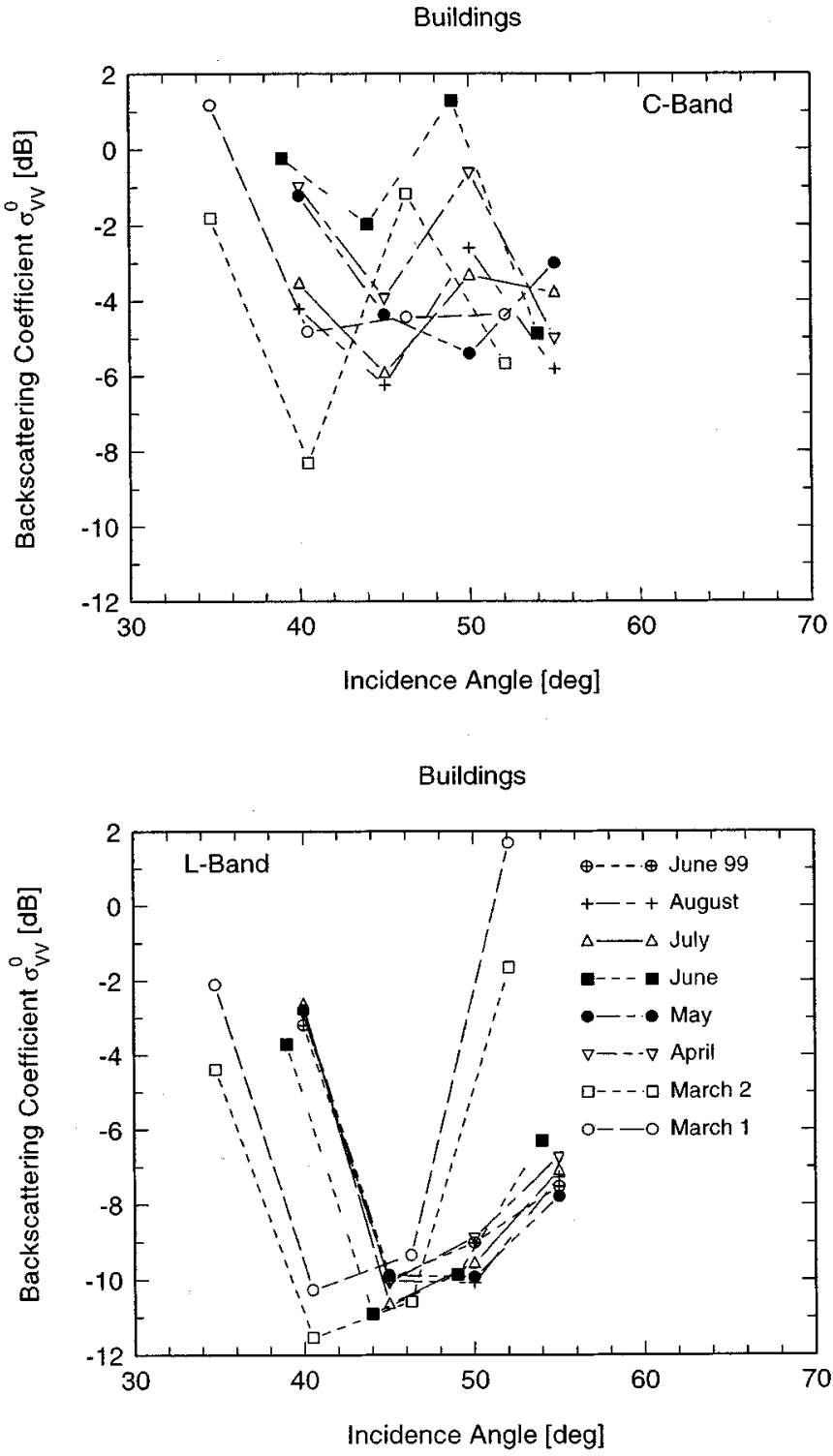

(c)

Fig. 3. (Continued.) (c) Backscattering coefficients of buildings at $\mathrm{C}$ - and $\mathrm{L}$-band, VV-polarization. The number of averaged pixels is larger than 525 .

quisitions is highly variable. At L-band, all curves have a similar shape, whereby the two March acquisitions are shifted by 3-5 deg toward smaller values of $\theta$ (because of a different illumination geometry, see Section II). Only at 55 deg (March: $52 \mathrm{deg}$ ), there is a large deviation between the data from March and from the other months. Since for given positions on the ground, the local incidence angles differ between the March scenes and the other images, the intensities backscattered from these positions are also different. The difference of the intensity levels between the two March data acquisitions which is almost constant over the whole incidence angle interval [Fig. 3(c)] compares well to the $2 \mathrm{~dB}$ difference observed for forests in Fig. 2(a).

The correlation coefficients, $\rho_{\mathrm{HHVV}}$ and the phase differences, $\phi_{\mathrm{HHVV}}$, between the HH- and VV-polarized channel are the parameters which are gained from a fully polarimetric SAR. The averages of $\phi_{\mathrm{HHVV}}$ as a function of incidence angle vary at maximum by $30 \mathrm{deg}$ (for object class "roads" at
L-band) between the different images (not shown). The largest difference of the average values of $\rho_{\mathrm{HHVV}}$ is observed for object class "roads," too, and is on the order of $\Delta \rho \approx 0.2$.

The backscattered intensities of the data sets from March and June 1998, which were acquired with slightly different illumination geometries, do in most cases not deviate much from the signatures observed in the reference images (1998: April, May, July, August; 1999: June). The only significant exception is observed for buildings at L-band at the incidence angle interval of 52.5-57.5 deg [Fig. 3(c)] as was already mentioned above. Since volume scattering is dominant for hedges, the sensitivity of the backscattered intensity to variations of the incidence angle is only weak over the SAR swath (incidence angle interval from $35 \mathrm{deg}$ to $60 \mathrm{deg}$ ) [Fig. 3(a)]. The observed sensitivity for roads is also comparatively weak [Fig. 3(b)], since the pixels marked as belonging to object class "roads" may include a contribution from the vegetation adjacent to the roads. In general, the sensitivity to the incidence angle is larger for surface scattering than for volume scattering, and it is larger for smoother surfaces. Hence, changes of the illumination geometry due to varying flight tracks should be kept smaller for surface scatterers than for volume scatterers. Urban environments, with their complex reflections mechanisms, are in general very sensitive to changes of the illumination geometry.

\section{B. Signature Variations}

The signature variations are due to speckle and texture. Texture is a measure of the intrinsic (or in-situ) variability of the backscattering coefficient (which would also be observed in the absence of speckle). It is hence related to spatial inhomogeneities of the scattering surface and/or volume, e.g., [10], [14]. The term "texture" as used here differs from its usual definition. Here, it denotes also spatial variations of the mean values of $\rho_{\mathrm{HHVV}}$ and $\phi_{\mathrm{HHVV}}$, and it refers both to the spatial variation of the backscattering coefficient within the area covered by a certain object (such as a hedge) as well as between different objects within one class. Texture is a function of frequency, incidence angle, polarization and spatial resolution of the imaging sensor. A useful parameter for the investigation of the inherent intensity variability caused by speckle and texture is the variance-to-squared-mean ratio (VMR) $\sigma_{P}^{2} /\langle P\rangle^{2}$, where $\sigma_{P}^{2}$ is the variance of the received power $P$, and \langle\rangle denotes the expected value. In this paper, the power is modeled by [15]

$$
P=[T\langle I\rangle+\langle n\rangle] S
$$

where $I$ is the power backscattered from a distributed target, $T$ is the texture random variable, $S$ is the speckle random variable, and $n$ is the system noise power.

Using the image model defined by (1), one obtains the VMR as a function of the effective number of independent looks, $L$, the power signal-to-noise ratio, $S N R$, and the variance of texture, $\sigma_{T}^{2}[15$, eq. 3]:

$$
\frac{\sigma_{P}^{2}}{\langle P\rangle^{2}}=\frac{1}{L}+\left(1+\frac{1}{L}\right)\left(1+\frac{1}{S N R}\right)^{-2} \sigma_{T}^{2} .
$$

For all images used, the VMR-values were evaluated as a function of the incidence angle by pooling all pixels belonging to 
TABLE II

(a) VMR RANGes of DifFerent ObJect Classes. OBSERVEd Minimum AND MaXimum VMRs out of THE 7 (8) IMAGES AT C-BAND (L-BAND), COMPRising

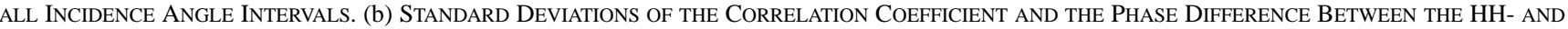
VV-Polarized Channel. Minimum and MaXimum Standard DeVIations ObSERVEd IN THE 7 (8) IMAGES AT C-BAND (L-BAND), COMPRISING ALL

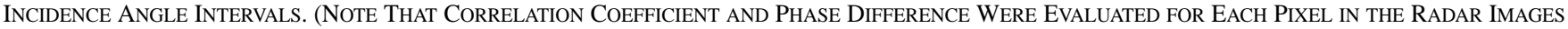
Before the Standard Deviations Were Determined For EACH ObJect Class as a Function of Incidence ANGLE)

\begin{tabular}{l|c|c|c|c|c|c}
\hline & \multicolumn{2}{|c|}{$\hat{\sigma}_{V V}^{0}$} & \multicolumn{2}{c|}{$\hat{\sigma}_{H H}^{0}$} & \multicolumn{2}{c}{$\hat{\sigma}_{H V}^{0}$} \\
\hline Hedges & C-Band & L-Band & C-Band & L-Band & C-Band & L-Band \\
\hline Roads & $0.19-0.45$ & $0.24-0.62$ & $0.16-0.50$ & $0.33-0.77$ & $0.20-0.46$ & $0.33-0.67$ \\
\hline Buildings & $0.2-1.0$ & $0.3-2.4$ & $0.2-1.5$ & $0.6-6.0$ & $0.4-2.1$ & $0.8-7.1$ \\
\hline Urban background & $1-100$ & $1-170$ & $2-90$ & $1-192$ & $1-70$ & $1-34$ \\
\hline Forest & $0.17-0.48$ & $0.19-0.45$ & $0.16-0.50$ & $0.21-0.42$ & $0.15-0.49$ & $0.18-0.37$ \\
\hline
\end{tabular}

(a)

\begin{tabular}{l|c|c|c|c}
\hline & \multicolumn{2}{|c|}{$\hat{\rho}_{H H V V}$} & \multicolumn{2}{c}{$\hat{\phi}_{H H V V}[\mathrm{deg}]$} \\
\hline Hedges & C-Band & L-Band & C-Band & L-Band \\
\hline Roads & $0.15-0.17$ & $0.16-0.20$ & $20.6-58.0$ & $50.6-85.6$ \\
\hline Buildings & $0.13-0.19$ & $0.15-0.23$ & $14.4-42.6$ & $29.0-63.2$ \\
\hline Urban background & $0.17-0.22$ & $0.18-0.21$ & $46.7-54.4$ & $67.7-83.2$ \\
\hline Forest & $0.14-0.18$ & $0.15-0.16$ & $24.3-41.9$ & $46.4-68.4$ \\
\hline
\end{tabular}

(b)

the selected object class within an incidence angle interval of 5 deg width. The results are listed in Table II(a). For the EMISAR covariance matrix product, the number of independent looks is $L \approx 11$ at C-band and $L \approx 9$ at L-band. If the backscattering coefficient of a distributed target in the EMISAR scene is constant (that is, $\sigma_{T}^{2}=0$ ), the VMR is 0.09 at C-band and 0.11 at L-band. The lowest VMR observed in the analyzed data set is 0.15 at C-band, and 0.18 at L-band (forest areas, cross-polarization, see Table II). Since the number of pixels from which the VMR was evaluated is larger than 170 in all cases, the estimation error of the VMR is negligible (see [15, appendix A]). Hence, the contribution of texture has to be considered for all object classes.

The values given in Table II(a) indicate that the signatures of the selected object classes are not homogenous, that is, natural variations of the backscattering coefficient (within one image) have to be considered. For change detection, it is hence important whether or not the natural spatial signature variation of an object remains temporally stable. By means of (2), it is possible to calculate the texture variance. In particular for roads, the signal-to-noise ratio in the EMISAR images can be as low as $10 \mathrm{~dB}$ at C-band which means that its influence on the VMR cannot be neglected. The VMR can be extremely large in the case of buildings and urban background due to the mixture of very different scattering mechanisms such as slightly rough surface scattering, on the one hand, and mirror and double bounce reflections, on the other hand. Note that (2) is strictly valid only for distributed targets, that is, for SAR resolution cells containing a larger number of randomly distributed scatterers, none of which is dominant. Urban areas are a mixture of pixels representing distributed targets and point targets (the latter are targets dominating the return in a resolution cell). The radar response from sufficiently large point targets (which appear often very bright in SAR images) does not exhibit speckle. In the analyzed scenes, pixels representing point targets make up only a very small fraction of the total area covered by villages but they have a very strong effect on the value obtained for the VMR because of the very large backscattered intensities relative to the distributed target responses.

The correlation coefficient, $\rho_{\mathrm{HHV}}$, and the phase difference, $\phi_{\mathrm{HHVV}}$, are not affected by texture provided that the length scales of the signature variations are larger than the multi-look resolution cell [16]. Hence, if theoretically predicted and observed variations of $\rho_{\mathrm{HHVV}}$ and $\phi_{\mathrm{HHVV}}$ are different, this indicates the influence of short-scale changes of the scattering characteristics. The standard deviation of $\phi_{\mathrm{HHVV}}$ depends on the correlation coefficient $\rho_{\mathrm{HHVV}}[10, \mathrm{p}$. 344], [16]. The estimate of the correlation coefficient is strongly biased for small "true" 
C-Band

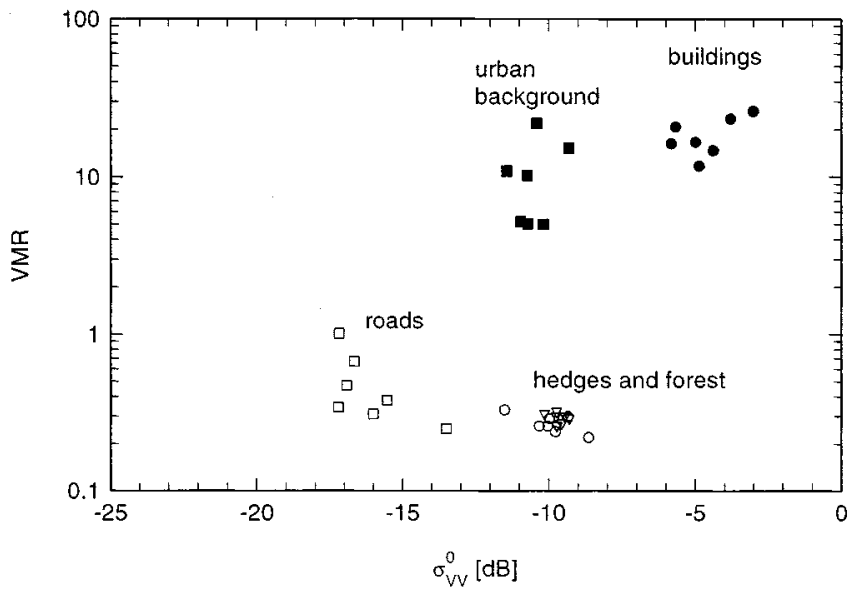

C-Band

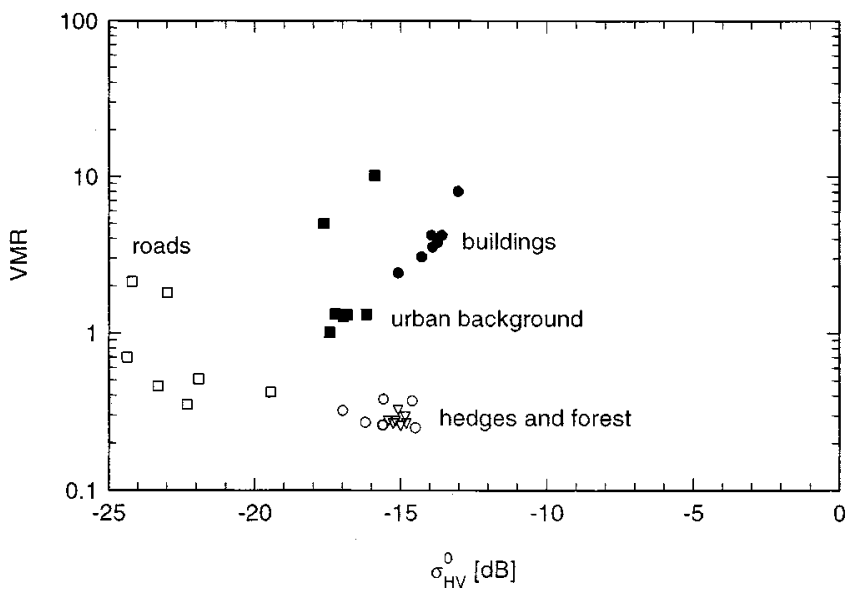

(a)
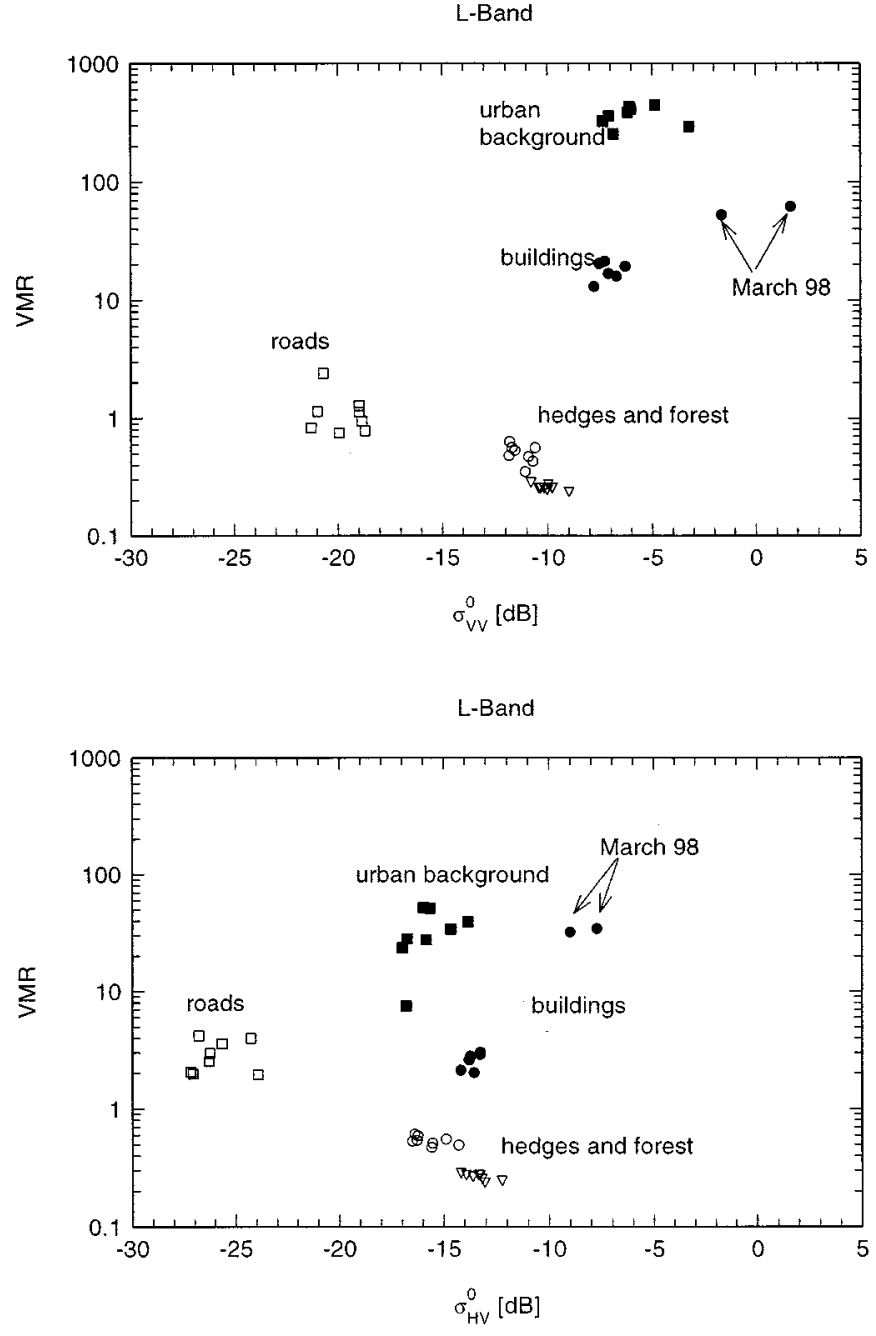

(b)

Fig. 4. Backscattering coefficients at VV-and HV-polarization and variance-to-squared-mean ratio (VMR) of different objects, obtained from 7 different EMISAR images. Open circles are used for hedges, open triangles for forest. The incidence angle range in the reference images is 52.5-57.5 deg. All pixels belonging to an object class within the incidence angle interval were pooled before calculating average backscattering coefficients and VMR's. Number of pixels are 2016 (hedges), 539 (roads), 31359 (forest), 1885 (buildings), and 13400 (urban background). (a) C-band. (b) L-band.

values of $\rho_{\mathrm{HHVV}}$ and a small number of looks [10, p. 345]; the estimated value is too large in this case. For example, if the number of looks is $L=9$, and the true correlation coefficient is $\rho_{\mathrm{HHVV}}=0.0$, one obtains $\hat{\rho}_{\mathrm{HHVV}}=0.3$ for the estimate. The correlation coefficients observed for the selected object classes vary between 0.3 and 0.7 , whereby the largest values were found for roads. From the probability distribution function given in [10, p. 345], the standard deviation of the correlation coefficient is calculated. Using $L=11$ at C-band, it lies between 0.11 and 0.13 , and with $L=9$ at L-band, between 0.12 and 0.15 . Here, a possible bias at low values of $\hat{\rho}_{\mathrm{HHVV}}$ was considered by taking $\rho_{\mathrm{HHVV}}=0.0$ as the lowest true value. Most of the minimum standard deviations of $\hat{\rho}_{\mathrm{HHVV}}$ listed in Table II(b) are smaller for C-band than for L-band, as is expected from theory. On the other hand, some of the maxima of the measured standard deviations of $\hat{\rho}_{\mathrm{HHVV}}$ are significantly larger than the theoretical values, in particular for buildings at $\mathrm{C}$-band. The standard deviation of $\phi_{\mathrm{HHVV}}$ due to speckle alone was evaluated numerically from the probability distribution function given in [10, p. 344]. It is $104 \mathrm{deg}$ for a "true" correlation coefficient of $\rho_{\mathrm{HHVV}}=0.0$, and $13.6 \mathrm{deg}$ for $\rho_{\mathrm{HHVV}}=0.7$. The standard deviations evaluated for the used images are within this range, a significant texture component cannot be identified. With regard to change detection, it is important to note that averages over single incidence angle intervals revealed estimates of $\rho_{\mathrm{HHVV}}$ as low as 0.4 ( 0.5 for roads) at C-band and between 0.3 and 0.4 at L-band for all object classes. This means that the phase difference variations due to speckle alone can be considerable. If two images are acquired at different times, the statistical uncertainty of $\hat{\phi}_{\mathrm{HHVV}}$ in each of the two images will in many cases be very large compared to the differences of average phases $\hat{\phi}_{\mathrm{HHVV}}$ between different object classes.

\section{Comparison of Signatures of Different Object Classes}

The average signatures of different objects together with their VMR's are shown in Figs. 4 and 5, focusing on a particular incidence angle interval in the reference scenes (it is considered that the same area lies in different angle intervals in March and June). It is worth to note that the average intensities of buildings and the urban background do not differ much at L-band. 

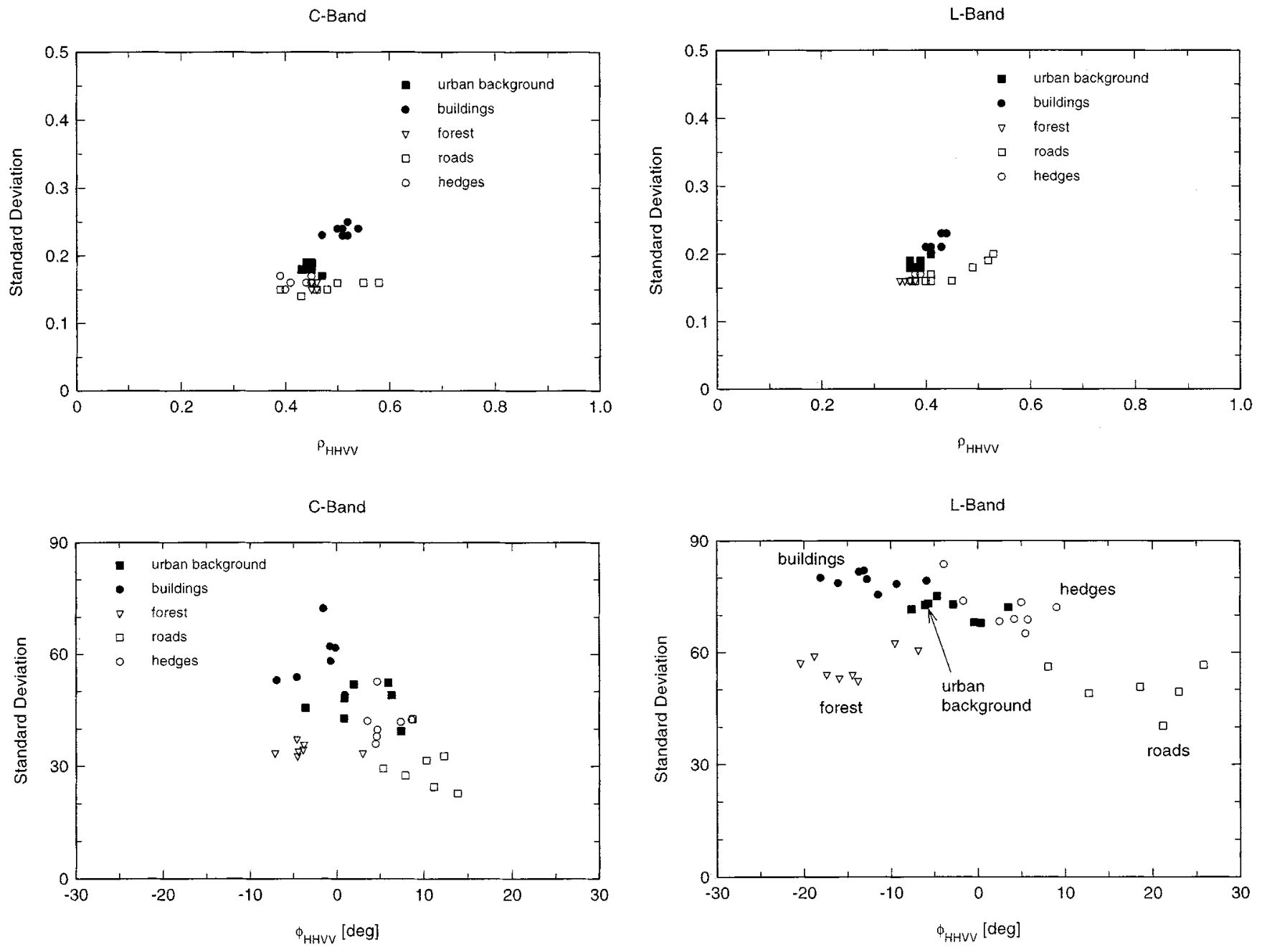

(a)

(b)

Fig. 5. Correlation coefficient and phase difference between $\mathrm{HH}$ - and VV-polarized channels, shown for different objects. The results were obtained in the same way as described in the caption of Fig. 4 after correlation coefficient and phase difference had been evaluated for each pixel. (a) C-band. (b) L-band.

At C-band, on the other hand, there is a difference of about 5 $\mathrm{dB}$ at VV-polarization [Fig. 4(a)]. This has to be considered for detecting the adding or removal of a building in an urban environment.

For change detection, the signature variations (due to speckle and temporal changes of backscattering characteristics) of both the object (which is added to or removed from the scene) and the "background" (which is covered by the added object or exposed after removal of the object) are of importance. A comprehensive analysis of the radar intensity contrast between all object classes relevant for change detection related to thematic mapping is beyond the scope of this paper. In an agricultural environment as it is typical for most parts of Denmark (and for large parts of Europe), object classes of interest for thematic mapping are often embedded in a background of agricultural crops. The polarimetric signatures of the latter (intensities at coand cross-polarization, correlation coefficient $\rho_{\mathrm{HHVV}}$ and phase difference $\phi_{\mathrm{HHVV}}$ ) are discussed in detail in [8]. The backscattered signatures of agricultural crops are highly variable over the period of one year. This requires that the image pair to be utilized for change detection is chosen such that the signatures of a certain agricultural crop are similar in both images. Moreover, since signatures vary considerably dependent on crop type, the intensity contrast between agricultural crops, on the one hand, and hedges, roads, and buildings, on the other hand, can only be maximized locally but not over the whole image.

Fig. 5 is an example where the phase difference and the correlation coefficient hardly contribute to the detection of changes because the observed differences between the object classes are statistically not significant, considering the magnitude of the standard deviation. If the objects of interest are embedded in a background of agricultural crops, the phase and correlation contrast between target and background may be large enough only at a few occasions dependent on crop type and season as can be deduced from the signatures presented by Skriver et al. [8].

The presented examples demonstrate that, all in all, the direct use of the correlation coefficient and the phase difference between the like-polarized channels is not "first choice" in a change detection algorithm for the object classes considered here but has a clearly lower priority. Therefore, the focus in the next section will be mainly on the measured intensities at the different polarizations. 


\section{TEMPORAL Signature VARIaTion}

This section deals with the temporal changes of object class signatures as it is observed in a sequence of multi-date SAR images. Besides "relevant" changes of the radar signature at a given position due to adding, removing or changing an object, the signature might vary because of the inherent natural variations of scattering characteristics and dielectric properties. These variations are modeled as texture and speckle. Technical factors to be considered are slightly varying imaging geometries, misregistration, and calibration errors which can be minimized by adequate measurement and data processing techniques. For the used data set, the magnitude of possible calibration variations is small but not negligible as is indicated by Fig. 2(a) and (b). The effect of misregistration is more difficult to assess because of the random signature variations between different images. A thorough inspection of the positions of the marked objects in the registered polarimetric images did not reveal any recognizable deviations. Hence, it can be assumed that "pseudo"-temporal signature variations of individual pixels due to inaccuracies in the registration are minimized as much as technically possible. The change of the local incidence angle at a given position is explicitly considered in the following subsections.

\section{A. Image Ratio and Correlation}

When dealing with speckled data, the ratio of two intensity images is better suited for change detection than the difference [5], [10, pp. 385-388]. For a speckled, but otherwise homogeneous region (texture $\sigma_{T}=0$ ), the estimated intensity ratio $Q=\left\langle I_{1}\right\rangle /\left\langle I_{2}\right\rangle$ is distributed according to [16], [10, p. 346]:

$$
P(Q)=\frac{\Gamma(2 L)}{\Gamma^{2}(L)} \frac{\left(1-|\rho|^{2}\right)^{L}(\gamma+Q) Q^{L-1} \gamma^{L}}{\left([\gamma+Q]^{2}-4|\rho|^{2} \gamma Q\right)^{L+1 / 2}}
$$

where $P$ is the probability density function (PDF), $\gamma$ is the true intensity ratio, $L$ is the number of looks, $\Gamma$ is the gamma function, and $\rho$ is the complex correlation coefficient between the two images which is estimated using [5]:

$$
\rho=\frac{\left\langle a_{1} a_{2}^{*}\right\rangle}{\sqrt{\left\langle\left|a_{1}\right|^{2}\right\rangle\left\langle\left|a_{2}\right|^{2}\right\rangle}}
$$

where $a_{1}$ and $a_{2}$ are the SAR complex amplitudes measured in image 1 and image 2 , and \langle\rangle indicates averaging over a window comprising an appropriate number of pixels. Equations (3) and (4) are still valid in the presence of texture, provided that the backscattering coefficient within one $L$-look pixel is constant [16], [17].

The correlation $\rho$ as defined by (4) is influenced by thermal noise, the antenna baseline separation, and temporal changes of the scattering characteristics (due to variations of the physical properties and/or positions of the scattering elements). The PDF given by (3) is useful for quantifying theoretically how often the ratio $Q$ exceeds a selected threshold [10, ch. 12.4]. For objects where the average intensity does not change between the two images, the probability of classifying inherent intensity variations as a real change is obtained for a given threshold if $\gamma$ is

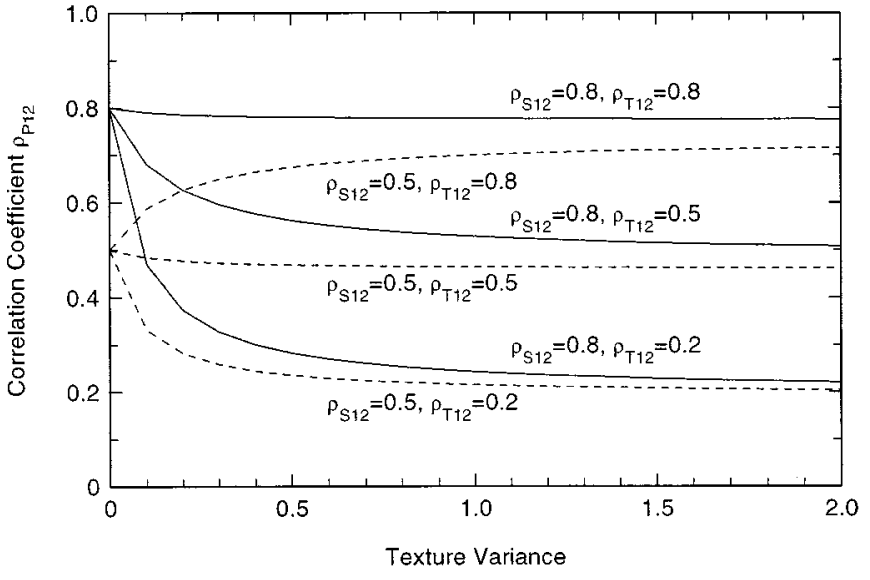

Fig. 6. The observed power correlation coefficient $\rho_{P 12}$ as a function of the texture variance $\sigma_{T}^{2}$ for different combinations of $\rho_{S 12}$ and $\rho_{T 12}$. The curves were evaluated using (5) assuming that the number of looks is $L=11, \sigma_{T 1}=$ $\sigma_{T 2}$, and the SNR is large.

set to one ("false alarm rate"). This requires that the calibration error is negligible.

Since the used data are in the covariance matrix format, only the correlation between the received power levels, $\rho_{P}$, can be calculated which in the absence of texture is $\rho_{P}=|\rho|^{2}$ [5], [18]. In its most general form, the correlation coefficient obtained from the intensity data is, using (1) as a model for the received power:

$$
=\frac{\rho_{S 12}\left(1+\frac{1}{S N R_{1}}\right)\left(1+\frac{1}{S N R_{2}}\right)+\left(L+\rho_{S 12}\right) \rho_{T 12} \sigma_{T 1} \sigma_{T 2}}{\sqrt{\sigma_{T 1}^{2}(L+1)+\left(1+\frac{1}{S N R_{1}}\right)^{2}} \sqrt{\sigma_{T 2}^{2}(L+1)+\left(1+\frac{1}{S N R_{2}}\right)^{2}}}
$$

where $L$ is the number of looks, $\rho_{S 12}=|\rho|^{2}, \rho_{T 12}$ is the correlation between the textures in images 1 and $2, \sigma_{T i}$ is the texture variance and $S N R_{i}$ is the signal-to-noise ratio in image $i$. If the signal-to-noise ratio is very large, and $\sigma_{T 1}=\sigma_{T 2},(5)$ is identical to (20) presented by Rignot and van Zyl [5]. It is noted that the correlation coefficient $\rho_{S 12}$ is independent of the number of looks [18]. Results for $\rho_{P 12}$ as a function of texture variance are shown in Fig. 6. From the figure and from (5) it is clear that the texture component has a strong influence on the magnitude of $\rho_{P 12}$, in particular, if $L$ is very large. In this case, $\rho_{P}$ cannot be directly used in (3). For the presented data set, the VMRs found for the different object classes [Table II(a)] reveal that the texture component is significant in most cases.

The coherence $\rho$ needed in (3) can be evaluated from the complex amplitude (magnitude and phase) image even if the signature variations include a texture contribution, provided that the length scales of the signature variations are larger than the SAR resolution cell [17]. However, complex amplitude data could not be utilized within the time frame of the project. The complex amplitude may be, in general, of interest for a pixel-to-pixel change detection approach. If the power magnitude within a resolution cell does not vary with time, but the effective phase (for example, as the result of a relative motion of the scatterers), the 
complex correlation $\rho$ between image 1 and image 2 reflects this change, but not the power correlation coefficient $\rho_{P 12}$. Since, on the other hand, such phase variations are rarely of importance in the type of change detection discussed here, the utilization of the intensity image is sufficient as long as the knowledge of coherence $\rho$ is not required explicitly in the change detection algorithm.

If the scatterer configuration within a resolution cell does not change as a function of time, the correlation $\rho_{S 12}$ is determined only by the system noise contribution and the antenna baseline (antenna separation). If a large signal-to-noise ratio is assumed, the difference between the flight tracks (which determines the baseline) is the dominant factor. The spatial baseline decorrelation coefficient is [15], [19]

$$
\rho_{B}=1-\frac{2(B \cos \theta) \Delta y \cos \theta}{\lambda R}
$$

where it is assumed that the baseline, $B$, is horizontal, $\Delta y$ is the spatial resolution in ground range, $R$ is the range distance to the target, $\theta$ is the incidence angle, and $\lambda$ is the radar wavelength. If the target lies on the plane to which the flight altitude $H$ is referenced, the range is $R=H / \cos \theta$. The baseline decorrelation depends strongly on the range position within the illuminated swath, and on the radar frequency. It is larger at near range and at shorter radar wavelengths.

The EMISAR navigational system permits to hold the airplane within a few meters [9]. Even if one takes variations in the aircraft attitude (pitch, yaw) into account, it can be assumed that the image pairs acquired with nominally identical illumination geometries (April-May, July-August) reveal correlation coefficients $\rho_{B}$ significantly larger than zero (in particular at far range). The reference images (April, May, July, August, and June 1999) were acquired at an altitude of $H=7600 \mathrm{~m}$. The single-look slant range resolution for the used EMISAR data is $2 \mathrm{~m}$. The ground range resolution is obtained from $2 / \sin \theta[\mathrm{m}]$. The critical baseline $\left(\rho_{B}=0\right)$ is $113 \mathrm{~m}$ at $\theta=35 \mathrm{deg}$ and $753 \mathrm{~m}$ at $\theta=60 \mathrm{deg}$ at C-band $(\lambda=0.057 \mathrm{~m})$. The corresponding numbers at L-band $(\lambda=0.25 \mathrm{~m})$ are $496 \mathrm{~m}$ and 3304 $\mathrm{m}$, respectively. The horizontal flight track shift of the March scenes relative to the reference images is about $1080 \mathrm{~m}$. Hence, the correlation $\rho_{P 12}$ between image pairs March-April is zero over the whole swath at C-band and over a stripe on the near range side of the swath at $\mathrm{L}$-band. If the baseline is vertical, the term $(B \cos \theta)$ has to be replaced by $(B \sin \theta)$. In this case, the minimum for the critical baseline is at $\theta=35 \mathrm{deg}$. It is 162 $\mathrm{m}$ at C-band and $681 \mathrm{~m}$ at L-band. The vertical offset for the June 1998 flight track is $277 \mathrm{~m}$, which means that for image pairs May-June and June-July, the speckle patterns at C-band are completely decorrelated in the near range part of the image.

From the images, an estimate of the correlation coefficient $\rho_{P 12}$ of the different object classes was evaluated using the relationship [10, p. 386]

$$
\hat{\rho}_{P 12}=\frac{\operatorname{var}\left\{P_{1}\right\}+\operatorname{var}\left\{P_{2}\right\}-\operatorname{var}\left\{P_{2}-P_{1}\right\}}{2 \sqrt{\operatorname{var}\left\{P_{1}\right\} \operatorname{var}\left\{P_{2}\right\}}}
$$

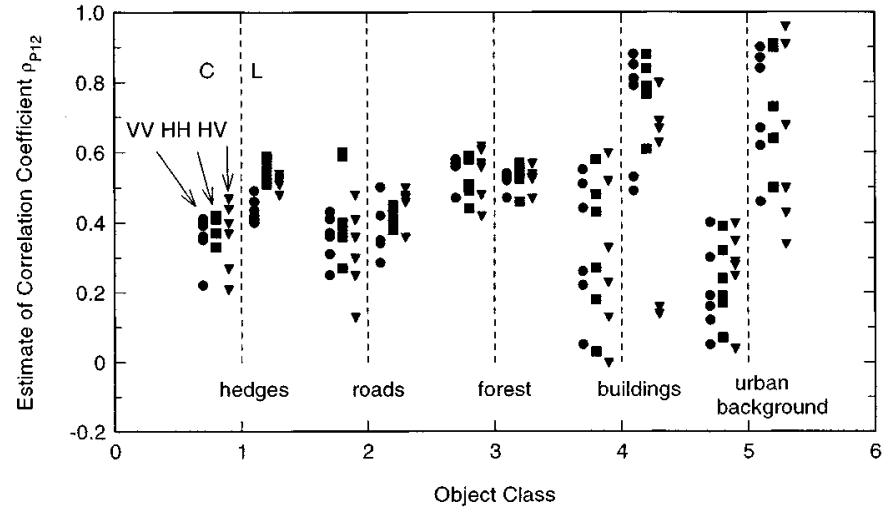

Fig. 7. Range of observed correlation coefficients $\rho_{P 12}$ for different object classes, evaluated using (6). The range intervals shown to the left of the dashed lines are for C-band, the ones to the right for L-band. The order of the depicted ranges is $\mathrm{VV}-, \mathrm{HH}-$, and $\mathrm{HV}$-polarization.

where $\operatorname{var}\{\}$ is the variance, and $P_{i}$ denotes the magnitudes of power of a group of adjacent pixels belonging to a certain object class in image $i$. If the variance of the difference $P_{2}-P_{1}$ is identical to the sum of the variances of $P_{1}$ and $P_{2}$, the intensities in image 1 and image 2 at a given position are uncorrelated $\left(\hat{\rho}_{P 12}=0\right)$. The estimate of the correlation coefficient $\rho_{P 12}$ was first computed as a function of incidence angle $\theta$ by pooling all pixels of an object class within a 5 deg wide incidence angle interval. However, a clear and systematic sensitivity of $\hat{\rho}_{P 12}$ to $\theta$ was not found. Hence, all pixels in the image belonging to the object class were used to calculate the variances in (7). The results are shown in Fig. 7. For the distributed targets (hedges, roads, and forests), the correlation can be as large as 0.6. The very low correlation coefficients for classes "buildings" and "urban background" at C-band are obtained for the image pairs March-April, since the shorter radar wavelength is more sensitive to changes in the illumination geometry. At L-band, on the other hand, the largest values of $\hat{\rho}_{P 12}$ are found for buildings and urban background which indicates that, at the longer wavelength, urban environments may reveal a more stable signature as a function of time than the distributed targets.

\section{B. Theoretical Prediction of Thresholds}

Having dealt with the theoretical fundamentals which have to be considered in the analysis of intensity changes in SAR images, one can now turn to the practical applicability of the mathematical models. In Fig. 8, theoretical histograms of the intensity ratio of forest areas which were evaluated using (3) are compared to the histograms measured for the six image pairs at C- and L-band separated by one month in time. The "measured" histograms were obtained by using all pixels belonging to the object class "forest" hence neglecting any sensitivity to the incidence angle which is justified in view of Fig. 2(a). The forest areas were selected as object class because they comprised a very large number of pixels. The agreement between theoretical and observed histograms is satisfactory at C-band and good at L-band, considering a slight uncertainty in the magnitude of the equivalent number of looks, and the presence of natural signature variations with unknown length scales. 


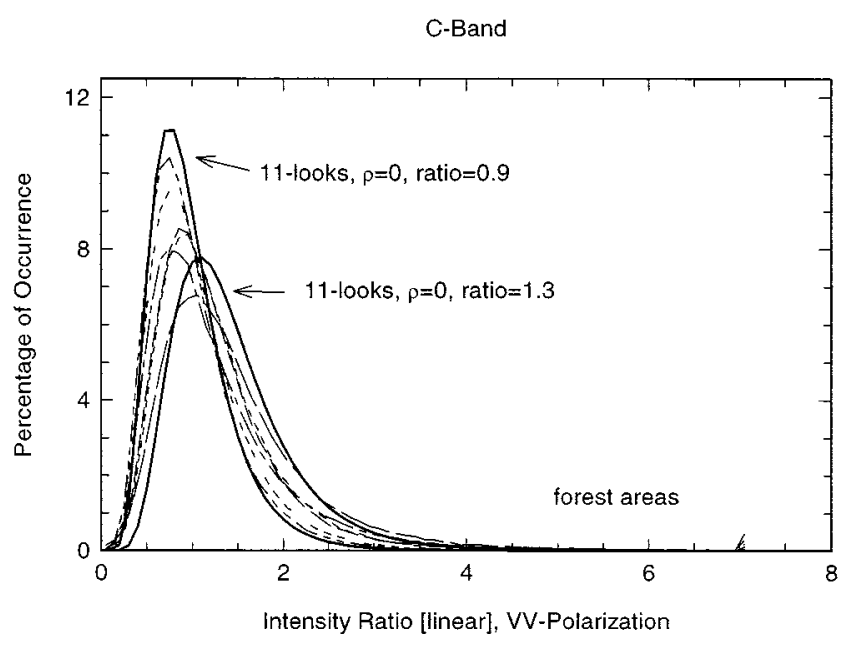

L-Band

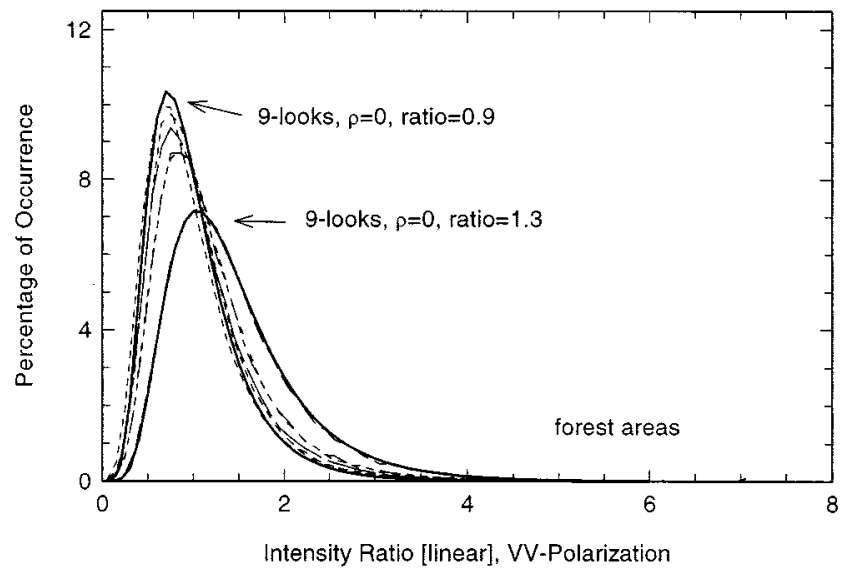

Fig. 8. Comparison of measured (dashed lines) and theoretical (solid lines) histograms of the intensity ratio at C- and L-band, VV-polarization, for forest areas. The measured histograms are for six image pairs with a temporal separation of one month, including all pixels belonging to object class "forest" over the whole swath width. The theoretical curves were evaluated using the minimum and the maximum of the observed average intensity ratios from Figs. 2 and 3.

The probability of classifying noise as a "real" signal is denoted the probability of false alarms, $P_{f a}$. This probability is given by [10, p. 387]

$$
P_{f a}=\operatorname{Pr} o b\{Q>T\}=\int_{T}^{\infty} P(Q) d Q .
$$

In this study, a probability of $P_{f a}=0.05$ is used, and the corresponding threshold is denoted as $T 5$. For this threshold, 5 per cent of the observed changes would be misinterpreted as real changes although they only reflect the variations due to speckle. In Fig. 9, thresholds are shown as a function of the correlation coefficient $\rho_{S 12}$ and the true intensity ratio, using (3). The threshold decreases with a decreasing intensity ratio and an increasing correlation coefficient.

How do the results compare to the thresholds which can be found from the intensity ratio histograms evaluated for the data set? The observed thresholds, $T 5_{o b s}$, were determined from the measured histograms for all image pairs separated by one

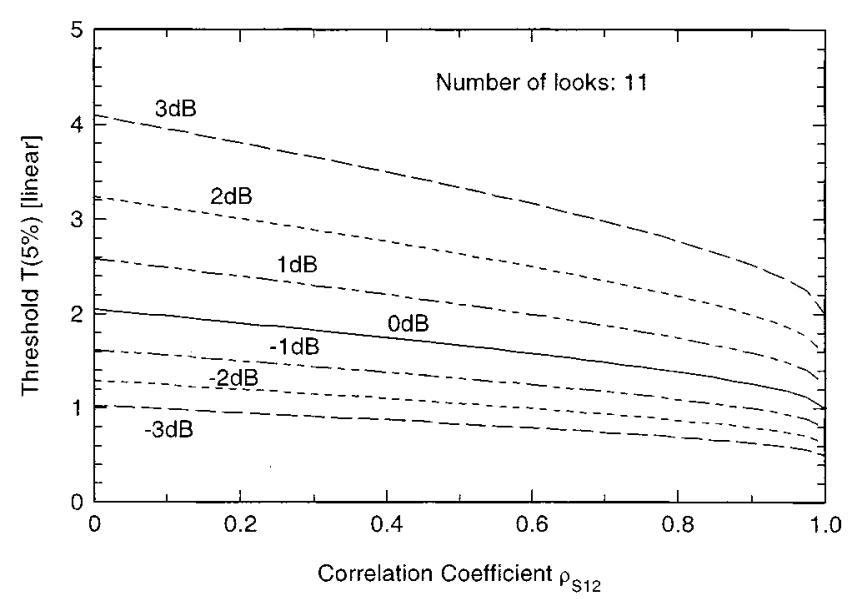

Fig. 9. Threshold above which 5\% of all pixels would be classified as real changes although the magnitude of their ratio is due to speckle.

month. The theoretical thresholds, $T 5_{\text {theor }}$, were obtained by evaluating $P(Q)$ using (3) with a value of $\rho=0$. Object classes "hedges" and "forest" were chosen for a more detailed analysis since their VMR-values were smallest. First, the investigation was carried out focusing on a particular incidence angle interval of $5 \mathrm{deg}$ in width in which a large number of pixels for each object class was available. As estimates of the true intensity ratio, $\gamma$, the observed average intensity ratios were taken. Then, average intensity ratios and histograms were evaluated from the data pooled over the entire swath width. It was found that the results did not differ significantly, and the conclusions which can be drawn from both cases are identical. The results obtained over the whole swath width are listed in Table III(a) and (b).

The values of the thresholds determined from the measured histograms reveal the tendency predicted by the curves shown in Fig. 9, namely that the magnitude of the threshold increases with the intensity ratio. All observed thresholds are larger than the predicted ones, whereby the differences between observed and theoretical values are smallest for forest areas at L-band (on average 0.1 ) and C-band (on average 0.3 ). The average differences for hedges are 0.47 at C-band and 0.85 at L-band. For roads, values of 0.86 at C-band and 1.1 at L-band were obtained. These differences increase if larger values for the correlation coefficient $\rho$ are chosen (which can not directly be determined from the intensity data, see above). The difference values were found for image pairs with a one-month period between data acquisitions as mentioned above. For the one-year pair at L-band, the corresponding values are 0.1 for forests, 0.7 for hedges, and 1.0 for roads.

The baseline decorrelation coefficient for most of the image pairs listed in Table III is significantly larger than zero. A small difference between observed and theoretical thresholds is obtained only if the theoretical thresholds are calculated assuming $\rho=0$. This means that the temporal decorrelation coefficient must be small in order to justify the assumption of $\rho=0$. For vegetated areas, this is plausible. For forests, for example, Askne et al. [20] observed coherencies, $\rho$, between 0.26 and 0.47 at C-band (the given values include both baseline and temporal effects) which means that $|\rho|^{2}$ lies in a range between 0.07 and 0.22 . Roads, on the other hand, should reveal larger 
TABLE III

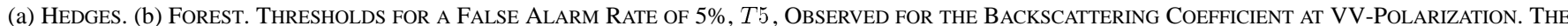
"TRUe" Intensity Ratio, $\gamma$, was Estimated Using the Data Depicted in Figs. 2(a) (Forest) and 3(a) (HedGeS). For the ObJeCt Classes Selected

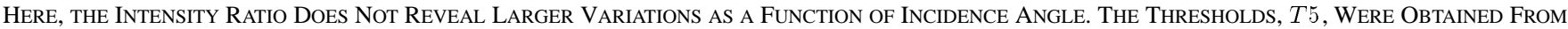
THE OBSERVEd Histograms of THE INTENSITY RATIO. THE DiFFERENCE BETWEEN OBSERVED AND THEORETICAL THRESHOLD IS $\Delta T=T 5-T 5_{t h e o r y}$.

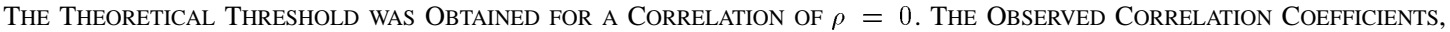
$\rho_{P 12}$ (WhICH INCLUDE THE TEXTURE COMPONENT) ARE AlSO SHOWN

\begin{tabular}{l|c|c|c|c|c|c|c|c}
\hline & \multicolumn{4}{|c|}{ C-Band } & \multicolumn{4}{c}{ L-Band } \\
\hline Image pair & $\gamma[\mathrm{dB}]$ & $T 5$ & $\Delta T$ & $\rho_{\text {observed }}$ & $\gamma \mathrm{dB}]$ & $T 5$ & $\Delta T$ & $\rho_{\text {observed }}$ \\
\hline March 1 - April & 0.4 & 2.55 & 0.30 & 0.41 & 0.2 & 3.35 & 1.02 & 0.42 \\
\hline March 2-April & 0.5 & 2.75 & 0.46 & 0.40 & -0.1 & 3.05 & 0.88 & 0.40 \\
\hline April - May & 2.6 & 4.45 & 0.72 & 0.39 & 1.4 & 4.45 & 1.39 & 0.46 \\
\hline May - June & -1.2 & 1.85 & 0.29 & 0.35 & 0.1 & 2.85 & 0.59 & 0.48 \\
\hline June - July & -1.6 & 1.95 & 0.54 & 0.2 & -0.7 & 2.25 & 0.37 & 0.41 \\
\hline July - August & 1.1 & 3.15 & 0.51 & 0.36 & 0.1 & 3.15 & 0.89 & 0.44 \\
\hline
\end{tabular}

(a)

\begin{tabular}{l|c|c|c|c|c|c|c|c}
\hline & \multicolumn{4}{|c|}{ C-Band } & \multicolumn{4}{c}{ L-Band } \\
\hline Image pair & $\gamma[\mathrm{dB}]$ & $T 5$ & $\Delta T$ & $\rho_{\text {observed }}$ & $\gamma[\mathrm{dB}]$ & $T 5$ & $\Delta T$ & $\rho_{\text {observed }}$ \\
\hline March 1 - April & 0.3 & 2.45 & 0.25 & 0.58 & 1.0 & 2.85 & 0.06 & 0.53 \\
\hline March 2 - April & 0.4 & 2.45 & 0.20 & 0.58 & -0.6 & 2.15 & 0.28 & 0.47 \\
\hline April - May & 0.3 & 2.75 & 0.55 & 0.47 & 0.2 & 2.45 & 0.12 & 0.53 \\
\hline May - June & -0.5 & 1.95 & 0.13 & 0.57 & 0.2 & 2.35 & 0.02 & 0.53 \\
\hline June - July & -0.1 & 2.15 & 0.14 & 0.57 & -0.1 & 2.25 & 0.08 & 0.52 \\
\hline July - August & 1.0 & 3.15 & 0.57 & 0.47 & -0.2 & 2.15 & 0.04 & 0.54 \\
\hline
\end{tabular}

(b)

values of coherency, but as already mentioned above, many of the pixels belonging to object class "roads" include signature contributions of the vegetation adjacent to the roads. In addition, the signal-to-noise ratio is lower compared to the other object classes.

Another important item to be considered is related to the length scales of the inherent signature variations. By means of Monte-Carlo simulations, Joughin et al. [16] obtained histograms of the intensity ratio for the case that the backscattering coefficient varies within a multi-look cell. As a consequence, the width of the distribution functions increases, as can be deduced from their results. The experimental observations presented here indicate that natural signature variations of hedges and of roads may occur on scales that are smaller than the $L$-look resolution cell (about $8 \mathrm{~m}$ wide for the EMISAR covariance matrix product).

For buildings, the situation is even more complicated. Besides partly extremely large values of the VMR [Table II(a)], the mean intensity ratio is characterized by strong local variations [which is reflected in Fig. 3(c)]. The number of pixels within a potential area of a more or less constant intensity ratio is too small to determine a statistically meaningful histogram. In order to find thresholds, histograms were evaluated using the pixels of class "buildings" distributed over the entire incidence angle interval. These histograms do not compare to theoretical predictions based on (3). Considering the observation that airborne SAR measurements from urban areas cannot be explained by models assuming constant scattering properties within the resolution cell [21], it may be reasonable to assume that urban signatures are in many cases also affected by inherent signature variations with length scales smaller than the effective spatial resolution of the imagery.

In Table III(a) and (b), the observed correlation coefficients $\rho_{\text {observed }}=\rho_{P 12}$ are listed as well. They indicate that texture between images, in contrast to speckle, is correlated to a certain degree.

\section{Temporal Signature Differences of the Selected Object Classes}

Histograms of pixel-to-pixel and object mean ratios (for $\rho_{\mathrm{HHVV}}$ and $\phi_{\mathrm{HHVV}}$ differences) were evaluated for image pairs 
TABLE IV

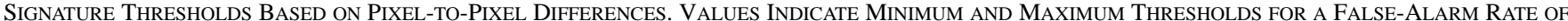
5\% Obtained From the Histograms of the 7 One-Month Image Pairs From 1998. The Data for All Objects Within One Object Class Were POOLED FOR THE CALCULATION OF THE HistogRams. THRESHOLDS $T l$ AND $T r$ FOR PHASE, $\phi_{\text {HHVV }}$, AND CORRELATION COEFFICIENT, $\rho_{\text {HHVV }}$, WeRE DETERMined FOR THE LEFT- AND Right-HAND Sides OF THE Histograms, So THAT $\int_{-\infty}^{T l} P(X) d X=0.025$ AND $\int_{T r}^{\infty} P(X) d X=0.025$

\begin{tabular}{l|c|c|c|c|c|c}
\hline & \multicolumn{3}{|c|}{ C-Band } & \multicolumn{3}{c}{ L-Band } \\
\hline & Hedges & Roads & Buildings & Hedges & Roads & Buildings \\
\hline$\sigma_{\mathrm{VV}}^{0}[\mathrm{~dB}]$ & $2.7,6.5$ & $2.2,5.7$ & $6.3,>10$ & $3.5,6.5$ & $2.9,6.9$ & $3.9,8.4$ \\
\hline$\sigma_{\mathrm{HH}}^{0}[\mathrm{~dB}]$ & $2.4,6.7$ & $2.4,7.5$ & $5.5,>10$ & $3.1,7.1$ & $2.9,6.6$ & $4.2,7.3$ \\
\hline$\sigma_{\mathrm{HV}}^{0}[\mathrm{~dB}]$ & $2.4,6.7$ & $2.4,7.5$ & $5.5,10$ & $3.7,6.8$ & $2.9,6.9$ & $4.1,8.7$ \\
\hline$\phi_{\mathrm{HHVV}}[\mathrm{deg}]$ & $-99,-81$ & $-81,-51$ & $-135,-99$ & $-147,-141$ & $-111,-75$ & $-147,-123$ \\
& 75,99 & 51,81 & 93,135 & 141,153 & 93,111 & 123,147 \\
\hline$\rho_{\mathrm{HHVV}}$ & $-0.42,-0.30$ & $-0.38,-0.26$ & $-0.50,-0.38$ & -0.38 & $-0.42,-0.30$ & $-0.38,-0.34$ \\
& $0.34,0.46$ & $0.30,0.42$ & $0.38,0.5$ & $0.38,0.42$ & $0.34,0.42$ & $0.34,0.46$ \\
\hline
\end{tabular}

TABLE V

Signature Thresholds Based on InHerent Signature Variability-ObJeCt Mean Values. Minumum and MaXimum Thresholds for a False Alarm Rate of 5\%. Note That the Number of Averaged Pixels Varies From Object to Object. See Also Comments Table IV

\begin{tabular}{l|c|c|c|c|c|c}
\hline & \multicolumn{3}{|c|}{ C-Band } & \multicolumn{3}{c}{ L-Band } \\
\hline & Hedges & Roads & Buildings & Hedges & Roads & Buildings \\
\hline$\sigma_{\mathrm{VV}}^{0}[\mathrm{~dB}]$ & $0.6,4.2$ & $0.2,4.5$ & $4.7,>10$ & $0.6,3.5$ & $0.6,5.4$ & $2.2,6.3$ \\
\hline$\sigma_{\mathrm{HH}}^{0}[\mathrm{~dB}]$ & $0.6,4.1$ & $-0.2,4.5$ & $4.1,>10$ & $0.2,3.9$ & $0.2,4.2$ & $2.2,5.2$ \\
\hline$\sigma_{\mathrm{HV}}^{0}[\mathrm{~dB}]$ & $0.6,4.1$ & $0.6,6.9$ & $3.5,>10$ & $0.6,3.1$ & $1.0,4.7$ & $1.9,6.0$ \\
\hline$\phi_{\mathrm{HHVV}}[\mathrm{deg}]$ & $-27,-15$ & $-21,-3$ & $-81,-45$ & $-39,-21$ & $-33,-15$ & $-81,-63$ \\
\hline$\rho_{\mathrm{HHVV}}$ & 15,27 & 15,21 & 63,69 & 21,45 & 21,33 & 57,99 \\
\hline$\ldots$ & $-0.14,-0.02$ & $-0.18,-0.02$ & $-0.34,-0.18$ & $-0.10,-0.06$ & $-0.22,-0.02$ & $-0.22,-0.14$ \\
$0.0 .06,0.18$ & $0.06,0.22$ & $0.18,0.34$ & $0.06,0.14$ & $0.06,0.30$ & $0.14,0.26$ \\
\hline
\end{tabular}

separated by one month by pooling the data over the entire image range. Ranges of the measured pixel-to-pixel thresholds obtained for the intensity ratios and the differences of $\rho_{\mathrm{HHVV}}$ and $\phi_{\mathrm{HHVV}}$ are listed in Table IV for object classes "hedges," "roads," and "buildings." The object mean ratio or difference was determined by averaging over the pixels of each object and subsequently computing the ratio or difference between the object mean in the one and in the other image. Since the objects are different in size, the mean values differ with regard to their statistical error because of the different number of samples. The obtained thresholds can be found in Table V. Examples for the histograms are shown in Fig. 10. The vertical lines indicate the minimum and maximum threshold for a change detection error less than 5 per cent, based on the histograms.

In order to keep the rate of false alarms low, the threshold for a pixel-to-pixel change detection has to be quite large in the case of buildings. In order to detect a real change, the threshold of the backscattering coefficient at VV-polarization, $\sigma_{\mathrm{VV}}^{0}$, for buildings at C-band, for example, should lie between $6.3 \mathrm{~dB}$ and larger than $10 \mathrm{~dB}$ to make sure that only a few detected events are due to inherent signal variations with the object itself being unchanged. If object averages are used, the corresponding minimum threshold decreases to $4.7 \mathrm{~dB}$, whereas the maximum remains at a level larger than $10 \mathrm{~dB}$. This can be explained by the fact that some of the buildings comprise less than 5 pixels which means that averaging does only slightly reduce the inherent signature variability.

Besides the number of looks, the thresholds, $T 5$, for forests, hedges, and roads depend on the average intensity ratio $\gamma$ and the correlation coefficient $\rho$. For the two latter object classes, the sensitivity of $T 5$ to the ratio $\gamma$ is plotted in Fig. 11 (whereby according to the results presented above it can be assumed that 
Roads

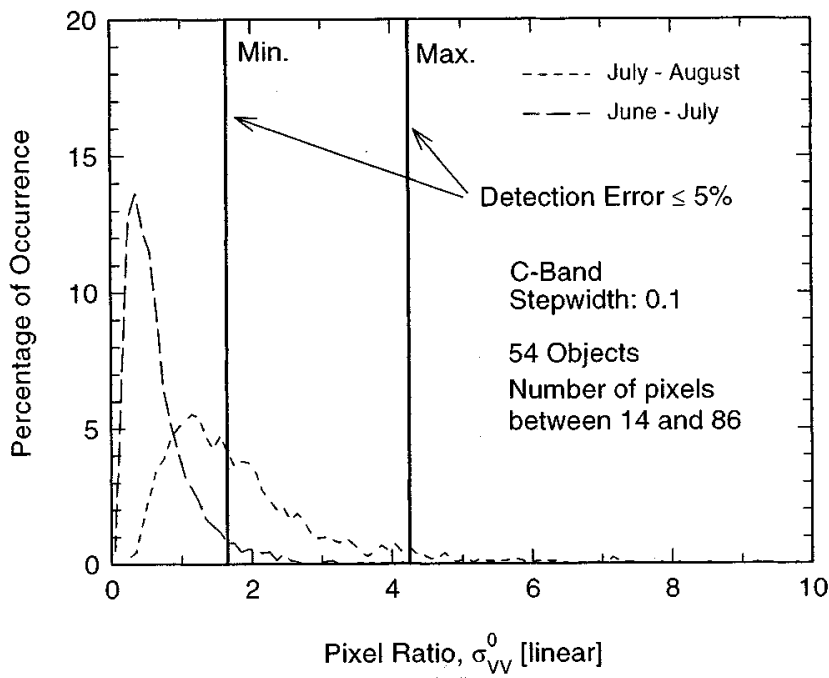

Roads

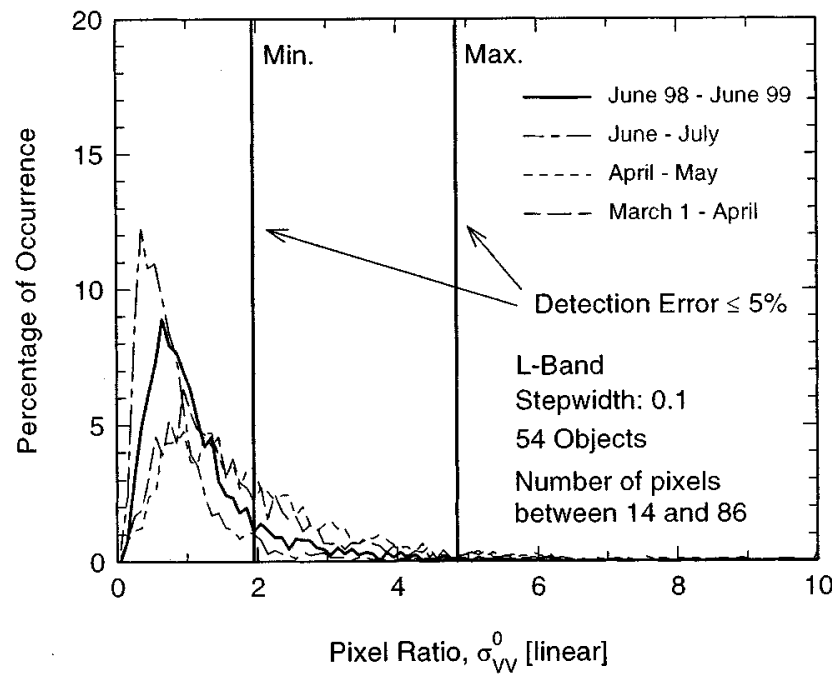

Fig. 10. Histograms of pixel-to-pixel intensity ratios at VV-polarization for buildings. Vertical lines indicate the threshold of $\sigma_{\mathrm{VV}}^{0}$ if changes have to be detected with an accuracy $\geq 95$ percent. The depicted histograms are for the minimum and maximum thresholds (if the same value is obtained from two histograms, both are shown).

$|\rho|^{2}$ is close to zero). The increase of the thresholds as a function of the average intensity ratio can be approximated by a linear function. The linear increase is in agreement with theoretical predictions represented by the solid lines in Fig. 11. The theoretical curve was calculated using (8) and (3), assuming that the correlation is $\rho=0$, and with a number of effective looks of $L=9$ at L-band, and of $L=11$ at C-band. The observed thresholds for the pixel-to-pixel approach are larger than the theoretical thresholds. The dashed lines reveal that the thresholds for roads are larger than for hedges (except for the pixel-to-pixel approach at L-band). It should be noted, however, that these differences between thresholds of different object classes are dependent on certain radar parameters (frequency and spatial resolution) which means that they should be determined for each new radar configuration. For practical applications it is worth to
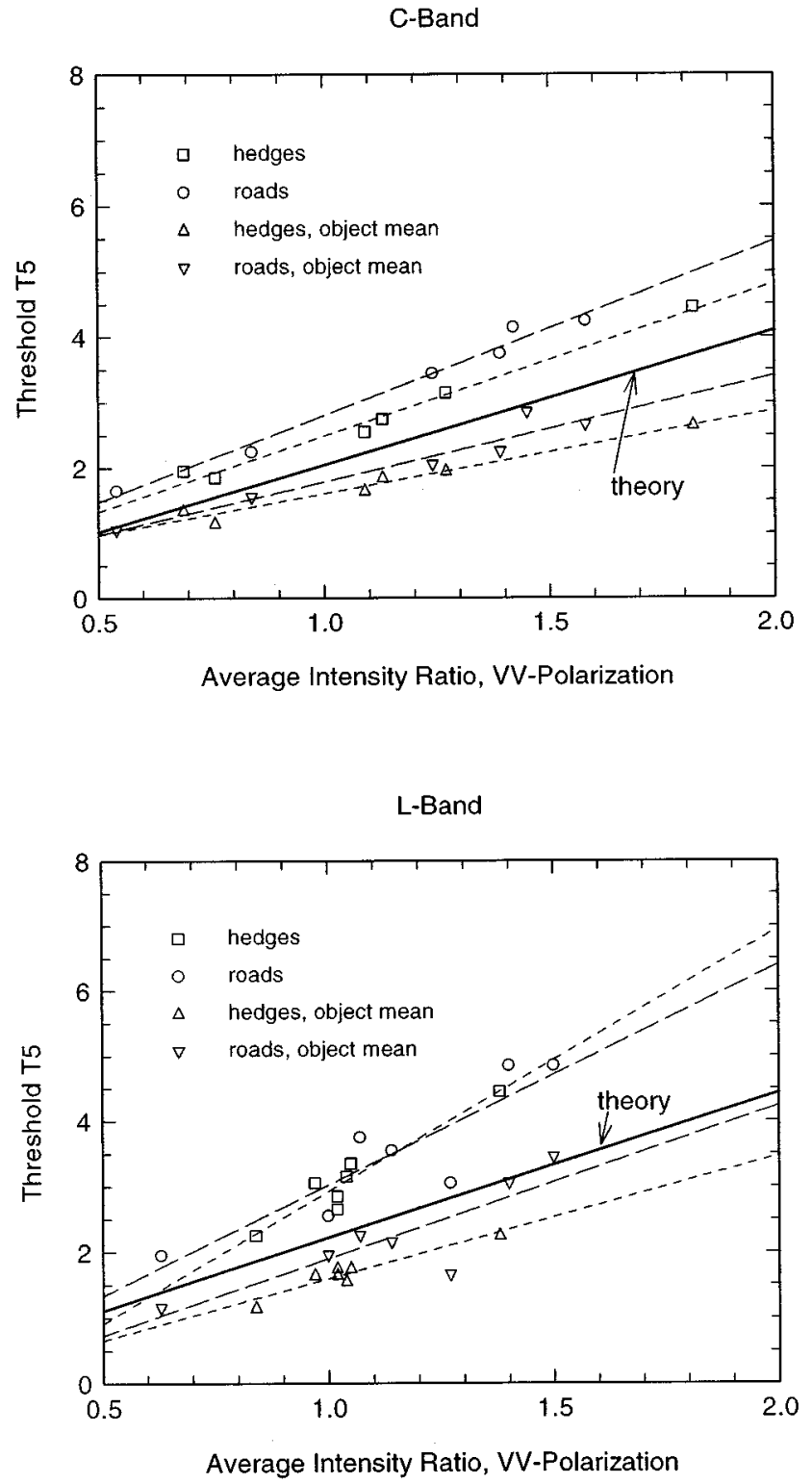

Fig. 11. Thresholds $T 5$ as a function of the average intensity ratio at VV-polarization for object classes hedges, roads, and buildings. Dashed lines are least-square fits. The theoretical results of a pixel-to-pixel approach are represented by the solid lines. Thresholds were determined for the observed histograms of the intensity ratio $Q$. Intensity ratio and threshold are given in linear scale.

note that the average intensity ratios for a given image pair differ between roads and hedges (Fig. 12) which means that thresholds may have to be determined separately for each object class. The thresholds of buildings decrease with an increase in the correlation coefficient $\rho_{P 12}$ as is shown in Fig. 13. The correlation coefficients are low for the image pairs March-April for which the deviation of the tracks flown during the measurements is largest.

\section{DISCUSSION}

The use of radar images in thematic mapping is attractive because radar imaging is not affected by cloud covers and light conditions. However, the interpretation of what is seen in the radar images is not always straightforward. For example, the 


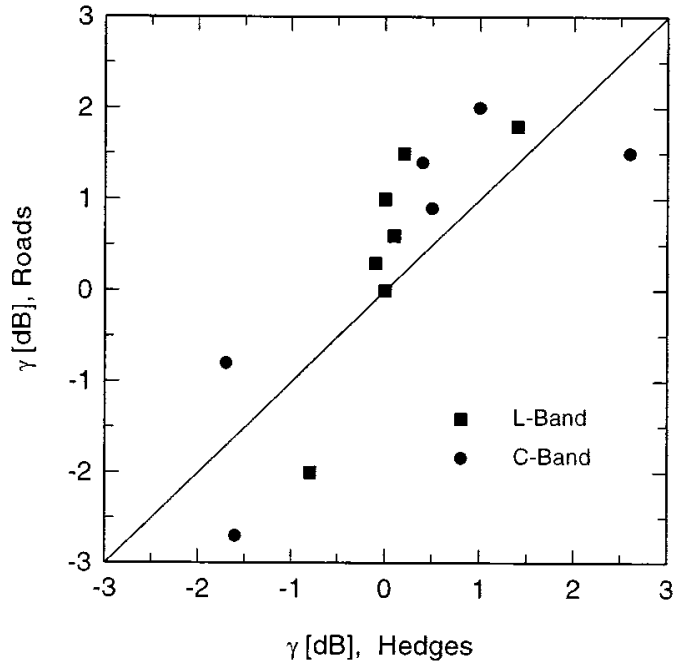

Fig. 12. Average intensity ratios $\gamma$ at VV-polarization at C- and L-band, compared for hedges and roads. Each point corresponds to the ratio obtained for one image pair. In order to use the same threshold for both hedges and roads, the point should lie on the solid line.

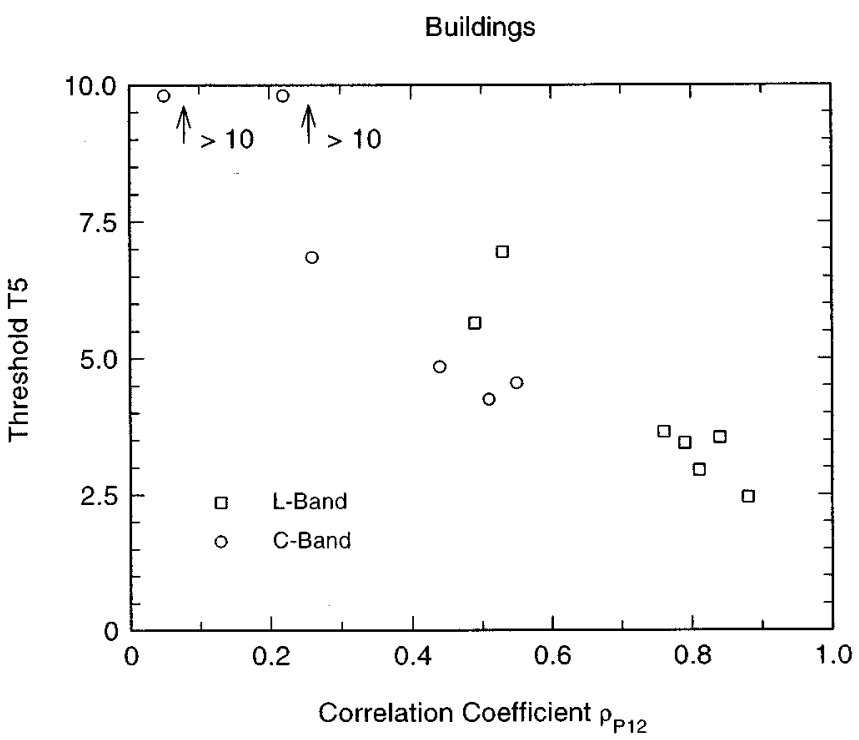

Fig. 13. Thresholds, $T 5$, at $\mathrm{C}$ - and L-band at VV-polarization as a function of the observed correlation coefficient $\rho_{P 12}$ for object class "buildings."

tree hedges stand out clearly in the radar image (Fig. 1), some roads, on the other hand, are more difficult to identify. Urban areas and also single buildings reveal often rather complicated signature patterns. Another disadvantage of applying radar images in thematic mapping is that the spatial resolution typically is much worse than for optical systems. For example, the aerial photography provided by KMS had a resolution of about 0.6 $\mathrm{m}$, whereas the single-look EMISAR images (scattering matrix format) have a resolution of $2 \mathrm{~m}$ (whereby one-look images are difficult to use because of speckle so that further averaging is needed). Hence, radar might be a useful complementary tool in thematic mapping, but, at least with present technology, it cannot replace optical systems.

In principle, the preprocessing required for change detection can be carried out without any source of information obtained by other sensors. This includes speckle reduction, edge detection, and segmentation whereby image processing techniques are utilized which are developed specifically for radar data. Speckle reduction techniques which do not blur the signatures of small or narrow objects require comparatively long run times on a computer [10], [11]. This is also valid for sophisticated segmentation techniques. In addition, some of these techniques require still further research and development. In the current work, the approach is to use the standard EMISAR images without any further processing in order to focus specifically on the influence of target signature variations. The images are directly compared to aerial photography. Note that also thematic maps of a sufficient scale could be used to this end. The Danish Survey and Cadastre, for example, provides GIS-data showing the position and shape of different objects. These vector layers can be directly combined with the registered EMISAR images. In the radar images, the pixels belonging to a certain object are identified and marked (this step would also be necessary in case of pre-segmented imagery). Any further processing steps may then be optimized for the different object classes of interest, e.g., by using class dependent thresholds for change detection.

Since for certain object classes, the length scales of inherent signature variations may be smaller than the SAR resolution cells, a theoretical prediction of the threshold for separating inherent variations from "real" changes is not always possible. Then, a better approach is to determine the threshold for a certain object class directly from the two images used for change detection. To this end, masks showing the position of different members of an object class can be used as explained above. A supervised method, for example, can be applied in which a comparatively small number of objects is used and regions of real changes are excluded. Alternatively, an unsupervised method is also possible in which the threshold is determined from a large number of objects. Compared to the total number of pixels belonging to one object class, the number of pixels affected by real changes will be very small and, hence, their influence on the estimated threshold is not critical.

If the intensity ratio of object means is chosen in order to find changes between two images acquired at different times, lower magnitudes can be used for the thresholds. However, this approach is meaningful only for objects which cover a larger area so that a sufficient number of pixels can be averaged. Objects belonging to one class may be different in size and, hence, the effective number of looks after averaging is different, too. Object mean values can be optimally utilized only in order to detect the removal of already existing objects. If new objects are added to a scene, their position has first to be identified in a ratio image obtained on a pixel-to-pixel basis or by moving windows. Changes of the "background" (usually natural or agricultural vegetation covers over most of the area) would also be visible, but could in many cases be easily identified and separated from the signals of smaller objects and linear features.

In general, an even better spatial resolution of the radar image product than the one achieved by EMISAR would be of advantage for the use in change detection. Technically, this is easier to realize at higher frequencies (C- and X-band). Single narrow objects of class "road," for example, could be located more precisely. Moreover, the signal-to-noise ratio of roads increases at 
shorter radar wavelengths. On the other hand, the inherent signature variations of urban areas would be more severe at higher radar frequencies, if the flight tracks of repeated data acquisitions deviate from one another. Also, because of the decreased size of a resolution cell, the number of scatterers inside the cell is reduced so that the speckle statistics might change.

A complete evaluation of the potential of SAR images for change detection for the objects considered in this paper, i.e., hedges, roads, and buildings, includes the assessment of the detection probability and the false alarm rate. The detection probability must be assessed both for objects which are added or removed during the period between two data acquisitions. The false alarm rate originates from two contributions which are the detection of changes when the objects are present in both acquisitions and when the objects are missing in both acquisitions, i.e., with only the background present. Such a complete evaluation was outside the scope of this paper. The assessment of the latter contribution to the false alarm rate, e.g., would require evaluation of a large number of different objects, such as the various crop types or different types of natural vegetation covers. Therefore, the only the contribution of the selected objects was considered.

\section{SUMMARY AND CONCLUSIONS}

If multi-channel imagery acquired by means of remote sensing is available for change detection, an interesting question is which combinations of the available channels are most useful. Another problem is to separate "real" changes from "inherent" variations of the image characteristics. Real changes are usually man-made (for example, the addition or removal of a building) and relevant for thematic mapping whereas inherent changes are not of interest for mapping agencies.

For this study, polarimetric signatures at C- and L-band acquired by an airborne SAR-system were available. The usefulness of different polarimetric parameters for change detection was analyzed. Inherent temporal variations in the polarimetric images were investigated in order to assess their influence on the change detection performance.

Inherent variations are caused by different factors:

- The physical properties of the imaged objects may change over time in a "natural" manner. The backscattering characteristics can vary significantly for certain object classes such as vegetation. Another effect is that a redistribution of scatterers within a resolution cell reduces or even erases the correlation of the speckle pattern between the two images.

- Technical effects such as errors in "operational" calibration and inaccuracies in the spatial registration of the multi-date images have to be considered.

- In practice, small deviations between the tracks flown when acquiring the image data are possible. These deviations cause speckle decorrelation, and they change the radar look angle at a given position on the ground. For example, an area viewed with different incidence angles $\theta$ might reveal differences in the backscattered intensities (since the backscattering coefficient of many surface types is a function of $\theta$ ) or might even change the scattering characteristics (e.g., if, at a given position, a specular reflection occurs only in one of the images).

In this study, specifically linear features (roads, tree hedges) and small objects (buildings) were considered which are of large interest for most mapping agencies. For comparison, object classes "urban background" and "forest" were included in the analysis. From the results of the study, the following conclusions can be drawn:

- The utilization of the phase difference $\phi_{\mathrm{HHVV}}$ improves the detection of changes only in very few cases (e.g., double bounce reflection for which $\phi_{\mathrm{HHV}} \approx 180 \mathrm{deg}$ versus surface scattering for which $\phi_{\mathrm{HHVV}} \approx 0 \mathrm{deg}$ ). The reason is that the statistical uncertainties of $\phi_{\mathrm{HHVV}}$ within one object class are usually large compared to differences of the mean values of $\phi_{\mathrm{HHV}}$ between different object classes. Also the correlation coefficient $\rho_{\mathrm{HHVV}}$ does not reveal usable systematic differences between the object classes. For the investigated classes, the utilization of the measured intensities has clearly a higher priority in practical applications compared to the other polarimetric parameters.

- For the linear features (roads and hedges), the intensity thresholds which have to be set in order to separate inherent signature variations from real changes do not differ significantly between C- and L-band. For buildings, the magnitudes of the thresholds are smaller at L-band than at C-band. A significant signature contrast between buildings and urban background, however, exists only at C-band at co-polarization.

- At larger incidence angles $(>35 \mathrm{deg})$, the measured intensities of the linear features are only weakly sensitive to variations of the incidence angle at a certain position. This means that images from different flight tracks can be combined provided that the distances between the tracks are within a given limit which depends on the scattering characteristics (volume scattering, smooth or rough surface scattering). For urban areas, the range of acceptable deviations is much smaller, in particular at shorter radar wavelengths.

- The magnitude of thresholds depends on the average intensity ratio and on the number of looks of the radar image. Because of inherent temporal variations of the average intensity ratio which are different for different object classes, change detection can be improved by fixing the thresholds separately for each class.

- Only for object class "forest," values of the thresholds can be evaluated theoretically independent of the observed texture (which indicates that scattering properties are locally constant). For object classes "hedges," "roads," and "buildings," the values of the thresholds have to be increased compared to the theoretical predictions. The interpretation is that inherent signature variations occur on scales smaller than a multi-look resolution cell. Urban areas reveal strong local variations of the average intensity ratio so that theoretical models which are based on the assumption of stationary signal variations cannot be used. 
This study has shown that radar images are useful complements for updating thematic maps. Furthermore, the study has provided hints for practical implementations of change detection techniques. Future research should address whether radar images of higher spatial resolution improve change detection, as well as focus on the simultaneous utilization of radar interferometry. Another interesting topic is to study the change detection performance for combinations of different polarimetric parameters.

\section{ACKNOWLEDGMENT}

The partners in the project group are from the Danish Survey and Cadastre (KMS), and from the Department of Planning (Technical University of Denmark). The authors would like to thank all members of the project group for the inspiring discussions during the regular meetings and for providing different kinds of assistance. It is also a pleasure to thank $\mathbf{J}$. Dall for his help regarding the processing of the EMISAR data.

\section{REFERENCES}

[1] R. G. White, "Change detection in SAR imagery," Int. J. Remote Sensing, vol. 12, no. 2, pp. 339-360, 1991.

[2] J. Cihlar, T. J. Pultz, and A. L. Gray, "Change detection with synthetic aperture radar," Int. J. Remote Sensing, vol. 13, no. 3, pp. 401-414, 1992.

[3] R. J. Dekker, "Speckle filtering in satellite SAR change detection imagery," Int. J. Remote Sensing, vol. 19, no. 6, pp. 1133-1146, 1998.

[4] M. Bao, "Backscattering change detection in SAR images using wavelet techniques," in Proc. Int. Geoscience Remote Sensing Symp. (IGARSS), vol. 2, 1999, pp. 910-912.

[5] E. J. M. Rignot and J. van Zyl, "Change detection techniques for ERS-1 SAR data," IEEE Trans. Geosci. Remote Sensing, vol. 31, pp. 896-906, Apr. 1993.

[6] J. D. Villasenor, D. R. Fatland, and L. D. Hinzman, "Change detection on Alaska's north slope using repeat-pass ERS-1 SAR images," IEEE Trans. Geosci. Remote Sensing, vol. 31, pp. 227-236, Jan. 1993.

[7] D. Weydahl, "Analysis of satellite SAR images for change detection over land areas," Norwegian Defence Research Establishment Publication 98/04 969, p. 234, 1998.

[8] H. Skriver, M. T. Svendsen, and A. G. Thomsen, "Multi-temporal C- and L-band polarimetric signatures of crops," IEEE Trans. Geosci. Remote Sensing, pt. II, vol. 37, pp. 2413-2429, May 1999.

[9] E. L. Christensen, N. Skou, J. Dall, K. W. Woelders, J. H. Jørgensen, J. Granholm, and S. N. Madsen, "EMISAR: An absolutely calibrated polarimetric L-and C-band SAR," IEEE Trans. Geosci. Remote Sensing, vol. 36, pp. 1852-1865, June 1998.

[10] C. J. Oliver and S. Quegan, Understanding SAR Images. Boston: Artech House, 1998.

[11] J. Schou and H. Skriver, "Restoration of polarimetric SAR images using simulated annealing," IEEE Trans. Geosci. Remote Sensing, vol. 39, pp. 2005-2016, Sept. 2001.

[12] W. Dierking, "EMISAR data processing: Registration of polarimetric images to DEM's generated from interferometric data," Dept. of Electromagnetic Systems, Technical Univ. of Denmark, Report R693, 2000.

[13] T. M. Lehmann, C. Gönner, and K. Spitzer, "Survey: Interpolation methods in medical image processing," IEEE Trans. Medical Imaging, vol. 18, pp. 1049-1075, Nov. 1999.
[14] W. Dierking, A. Carlström, and L. M. H. Ulander, "The effect of inhomogeneous roughness on radar backscattering from slightly deformed sea ice," IEEE Trans. Geosci. Remote Sensing, vol. 35, pp. 147-159, Jan. 1997.

[15] E. Rignot and R. Kwok, "Characterization of spatial statistics of distributed targets in SAR data," Int. J. Remote Sensing, vol. 14, no. 2, pp. 345-363, 1993

[16] I. R. Joughin, D. P. Winebrenner, and D. B. Percival, "Probability density functions for multilook polarimetric signatures," IEEE Trans. Geosci. Remote Sensing, vol. 32, pp. 562-574, Mar. 1994.

[17] R. Touzi, A. Lopes, J. Bruniquel, and P. W. Vachon, "Coherence estimation for SAR imagery," IEEE Trans. Geosci. Remote Sensing, vol. 37, pp. 562-574, Jan. 1999.

[18] J. S. Lee, K. W. Hoppel, S. A. Mango, and A. R. Miller, "Intensity and phase statistics of multilook polarimetric and interferometric SAR imagery," IEEE Trans. Geosci. Remote Sensing, vol. 32, pp. 1017-1027, May 1994.

[19] H. Zebker and J. Villasenaor, "Decorrelation in interferometric radar echoes," IEEE Trans. Geosci. Remote Sensing, vol. 30, pp. 950-959, May 1992.

[20] J. Askne, P. B. G. Dammert, L. M. H. Ulander, and G. Smith, "C-band repeat-pass interferometric SAR observations of the forest," IEEE Trans. Geosci. Remote Sensing, vol. 35, pp. 25-35, Jan. 1997.

[21] J. A. Tough, D. Blacknell, and S. Quegan, "A statistical description of polarimetric and interferometric synthetic aperture radar data," in Proc. Roy. Soc. London A., vol. 449, 1995, pp. 567-589.

Wolfgang Dierking received the diploma degree in geophysics from the University of Hamburg, Germany, and the Ph.D. degree in physics from the University of Bremen, Germany, in 1985 and 1989, respectively. His Ph.D. project dealt with radar remote sensing of the ocean.

From 1990 to 1993, he was with the Alfred-Wegener-Institute for Polar and Marine Research, Bremerhaven, Germany, where he worked on microwave remote sensing of ice and snow and on airborne measurements of the sea ice surface topography by means of laser altimetry. From 1993 to 1995, he joined the Remote Sensing Group at Chalmers University of Technology, Göteborg, Sweden, where he focused on scatterometry and microwave scatter modeling with applications to sea ice monitoring. Since 1996, he has been with the Department of Electromagnetic Systems, Technical University of Denmark, Lyngby, Denmark. His current research deals with the utilization of polarimetric and interferometric SAR data in geoscientific research. He has participated in several land-based, ship-based, and airborne field studies related to remote sensing projects.

Henning Skriver received the M.Sc. and Ph.D. degrees, both in electrical engineering, from the Technical University of Denmark, Lyngby, Denmark, in 1983 and 1989 , respectively

He has been with the Electromagnetics Institute (EMI), now Ørsted•DTU Department, Technical University of Denmark, Lyngby, since 1983, where he is now an Associate Professor. His work has primarily been concerned with various topics related to the utilization of SAR data for different applications. From 1983 to 1992, his main area of interest was retrieval of sea ice parameters from SAR data, including SAR data from ERS-1. Since 1992, he has covered different aspects of land applications of SAR data, such as forestry in the MAESTRO-1 project, agricultural and environmental applications using both satellite SAR data and data from the Danish airborne polarimetric SAR, EMISAR. His interests also include various methods for processing of SAR-data, such as SAR image simulation, SAR image filtering, speckle statistics, texture analysis, segmentation, calibration, and polarimetric analysis. He has currently been project manager of a project concerning the use of SAR data for cartographic mapping. 